\title{
Article
}

\section{CuI-Catalyzed Coupling Reactions of 4-Iodopyrazoles and Alcohols: Application toward Withasomnine and Homologs}

\author{
Yoshihide Usami *®D, Yumika Kubo, Toshiki Takagaki, Nao Kuroiwa, Jun Ono, Kohei Nishikawa, Ayaka Nakamizu, \\ Yuya Tatsui, Shinya Harusawa, Noboru Hayama and Hiroki Yoneyama
}

check for

updates

Citation: Usami, Y.; Kubo, Y.;

Takagaki, T.; Kuroiwa, N.; Ono, J.;

Nishikawa, K.; Nakamizu, A.; Tatsui,

Y.; Harusawa, S.; Hayama, N.; et al.

CuI-Catalyzed Coupling Reactions of

4-Iodopyrazoles and Alcohols:

Application toward Withasomnine

and Homologs. Molecules 2021, 26,

3370. https://doi.org/10.3390/

molecules 26113370

Academic Editor: Mohammad Alam

Received: 10 May 2021

Accepted: 31 May 2021

Published: 2 June 2021

Publisher's Note: MDPI stays neutral with regard to jurisdictional claims in published maps and institutional affiliations.

Copyright: (c) 2021 by the authors. Licensee MDPI, Basel, Switzerland. This article is an open access article distributed under the terms and conditions of the Creative Commons Attribution (CC BY) license (https:/ / creativecommons.org/licenses/by/ $4.0 /)$.
Department of Pharmaceutical Organic Chemistry, Osaka University of Pharmaceutical Sciences, 4-20-1 Nasahara, Takatsuki, Osaka 569-1094, Japan; e12343@gap.oups.ac.jp (Y.K.); e16442@gap.oups.ac.jp (T.T.); e16332@gap.oups.ac.jp (N.K.); e15439@gap.oups.ac.jp (J.O.); e15637@gap.oups.ac.jp (K.N.); e14519@gap.oups.ac.jp (A.N.); e18902@gap.oups.ac.jp (Y.T.); harusawa@gly.oups.ac.jp (S.H.); hayama@gly.oups.ac.jp (N.H.); yoneyama@gly.oups.ac.jp (H.Y.)

* Correspondence: usami@gly.oups.ac.jp; Tel.: +81-726-90-1087

Abstract: The direct 4-alkoxylation of 4-iodo- $1 H$-pyrazoles with alcohols was achieved by a CuIcatalyzed coupling protocol. The optimal reaction conditions employed excess alcohol and potassium $t$-butoxide (2 equiv) in the presence of $\mathrm{CuI} \mathrm{(20} \mathrm{mol \% )} \mathrm{and} \mathrm{3,4,7,8-tetramethyl-1,10-phenanthroline}$ $(20 \mathrm{~mol} \%)$ at $130{ }^{\circ} \mathrm{C}$ for $1 \mathrm{~h}$ under microwave irradiation. The present method was efficiently applied to the synthesis of withasomnine and its six- and seven-membered cyclic homologs.

Keywords: synthesis; 4-alkoxypyrazole; CuI; coupling reaction; microwave; withasomnine; homologue

\section{Introduction}

Owing to their diverse bioactivities, both natural and synthetic pyrazoles and pyrazolefused heterocycles have been widely exploited as pharmaceutical or pesticide active ingredients [1-4]. Therefore, the efficient synthesis of substituted pyrazoles possessing characteristic functionalities at specific positions is an important objective in organic and medicinal chemistry, as well as in drug discovery. In this context, we recently reported palladium- or copper-catalyzed C-N coupling reactions at the C-4 positions of pyrazoles [5]. Although metal-catalyzed $\mathrm{C}-\mathrm{O}$ coupling reactions have been widely reported, owing to their wide-ranging potentials [6-12], the direct $\mathrm{C} 4-\mathrm{O}$-functionalization of pyrazoles has not yet been studied satisfactorily $[13,14]$ despite the important bioactivities that have been demonstrated for several 4-alkoxypyrazoles, as presented in Figure 1.

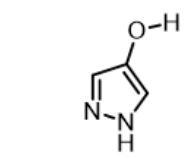

4-hydroxypyrazole (metabolite of pyrazole)

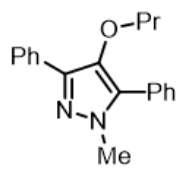

fungicide

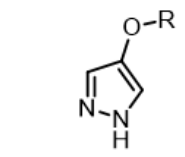

$\mathrm{R}=\mathrm{Me}, \mathrm{Et}, n-\mathrm{Pr}$, isoPr

LAD inhibitors

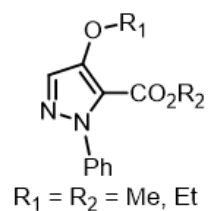
antiinflammatory, analgesic activities antipyretic, sedative,

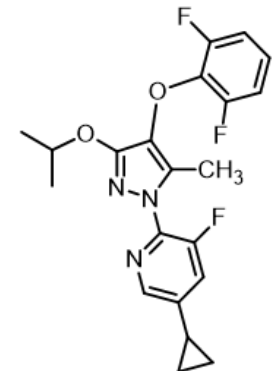

human DHODH inhibitor
Figure 1. Bioactive 4-alkoxypyrazoles. 
4-Hyroxypyrazole, a metabolite of pyrazole, exhibits various biological activities such as anti-inflammatory, antipyretic, antitumor, antifungal effects [15]. 4-Methoxy-, 4-ethoxy, 4-n-propoxy-, and 4-isopropoxypyrazoles have been reported to inhibit liver alcohol dehydrogenase (LAD) in humans, rats, and horses [16]. In particular, 4-ethoxypyrazole and 4-propyloxypyrazole have been recognized as a cytochrome P-450 inducer [17] and cytochrome P450 2E1 inhibitor, respectively [18]. Two C4-O-functionalized pyrazoles have been patented: 1-methyl-3,5-diphenyl-4-propoxypyrazole, as a fungicide [19], and the alkyl (4-alkoxy-1-phenyl)pyrazolylcarboxylates, which possess antipyretic, sedative, antiinflammatory, and analgesic activities [20]. A compound bearing a 4-(2,4-difluorophenyl)oxy group was revealed as a human dihydroorotate dehydrogenase (DHODH) inhibitor [21].

4-Allyloxy-1H-1-tritylpyrazole 4a (Scheme 1), derived from 4-iodopyrazole (1), played a key role as a versatile intermediate in our previous studies for the total synthesis of the pyrazole alkaloid, withasomnine (7) [22,23], and its six-membered homolog 11 [23-27], which were reported to exhibit COX-2 inhibitory activities $[23,27,28]$. Compound $4 \mathbf{a}$ was also extensively utilized as an important intermediate for the construction of new pyrazolefused heterobicyclic molecules 9 via ring-closing metathesis (RCM) (Scheme 1) [29-31]. However, the synthesis of compound 4 a requires six steps from commercially available pyrazole, through 4-iodopyrazole (1), 4-iodo- $1 H$-1-tritylpyrazole (2a), and aldehyde 3 . If direct $O$-allylation from 1 could be realized, the synthesis of several synthetic targets would be remarkably shortened. Thus, we focused our attention on the direct $\mathrm{C}-\mathrm{O}$ coupling reactions of 1 , based on our prior report of the $\mathrm{C}-\mathrm{N}$ couplings of 4-halopyrazoles [5]. Herein, we disclose $\mathrm{CuI}$-catalyzed coupling reactions of 4-iodo- $1 \mathrm{H}$-pyrazoles and alcohols. Furthermore, the developed method was applied to improve the synthesis of withasomnine and its homologs containing six- or seven-membered ring systems.

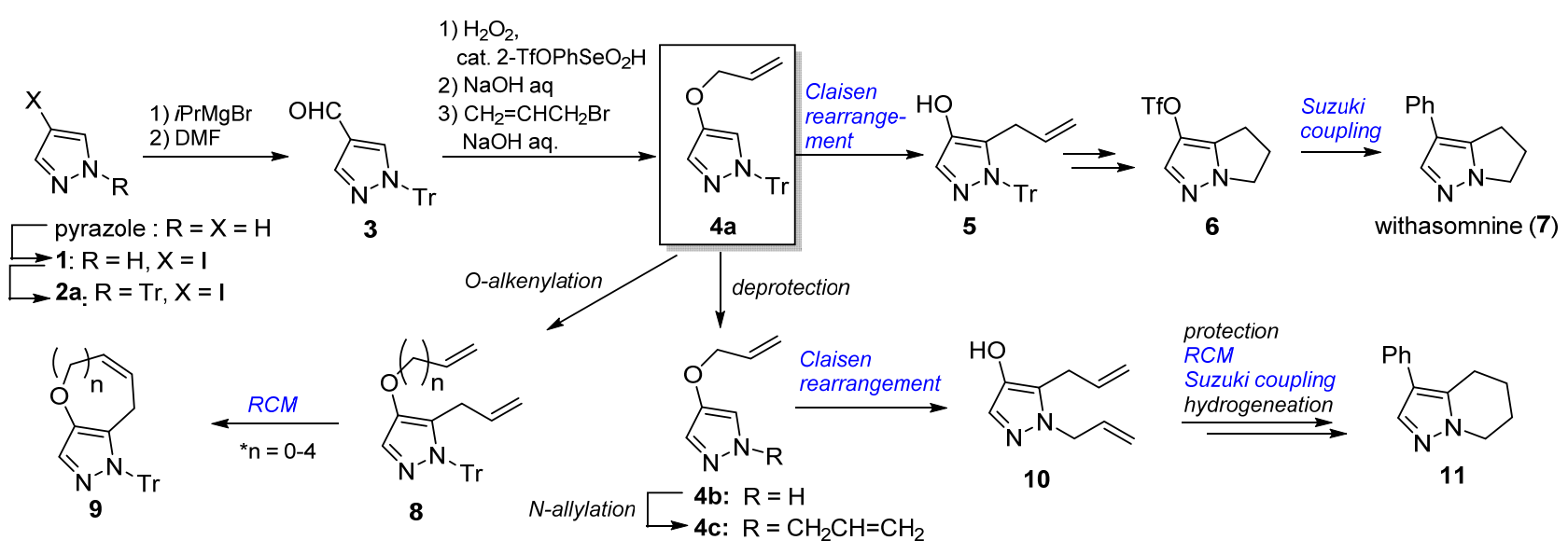

Scheme 1. Versatile intermediate 4-allyloxy- $1 H$-tritylpyrazole (4a), developed in our previous works (adapt from references [22-24,29-31]).

\section{Results and Discussions}

\subsection{Investigation of 4-O-Allylation of 4-Iodopyrazole}

Initially, we attempted the $\mathrm{Pd}(\mathrm{dba})_{2}$-catalyzed reaction between $2 \mathrm{a}$ and allyl alcohol (2 equivalent (equiv)) in the presence of $t$ BuDavePhos as a ligand and potassium tert-butoxide $\left({ }^{t} \mathrm{BuOK}\right)$ as a base under the reaction conditions in our previous report [5]; however, none of the desired coupling product was obtained (Table 1, entry 1). The corresponding 4-bromo- $1 \mathrm{H}$-1-tritylpyrazole was not effective in the palladium-catalyzed coupling reaction. Then, the CuI-catalyzed reaction between 4-iodo- $1 \mathrm{H}$-1-tritylpyrazole 2a and allyl alcohol was examined; the results are summarized in Table 1. All reactions were performed using $\mathbf{2 a}(50 \mathrm{mg})$ in a solvent $(2.0 \mathrm{~mL})$. In the presence of ligand 2-isobutyroylcyclo-hexanone (L2) or 1,10-phenanthroline (L3) in N,N-dimethylformamide (DMF) [5], reactions of $\mathbf{2 a}$ and allyl alcohol (2 equiv) did not afford $\mathbf{4 a}$ (entries 2 and 3 , respectively). However, when allyl alcohol was used as a solvent for this reaction 
with $\mathbf{L} 3$ at $100{ }^{\circ} \mathrm{C}$ overnight, the desired C4-O-allylation product 4a was obtained in 51\% yield (entry 4). Next, microwave (MW) assistance was applied to reduce the reaction time (entries 5-9). In these experiments, the reaction time was fixed at $1 \mathrm{~h}$ and the ligand was changed to 3,4,7,8-tetramethyl-1,10-phenanthroline (L4). From entry 6, the optimum reaction temperature was determined to be $130{ }^{\circ} \mathrm{C}$, giving $4 \mathrm{a}$ in $66 \%$ yield. At $160{ }^{\circ} \mathrm{C}$, the reaction mixture turned black with a poor yield of $4 a(16 \%$, entry 8$)$. In addition, shortening the reaction time $(30 \mathrm{~min}$ ) or reducing the amount of $\mathrm{CuI}$ to $10 \mathrm{~mol} \%$ afforded 4a in lower yields (entry 7:24\%; entry 9:37\%, respectively). Based on these results, the optimum conditions obtained in entry 6 were applied in the following coupling reactions of 4-iodopyrazoles with various alcohols.

Table 1. Optimization of CuI-catalyzed reaction between 4-iodo-1H-1-tritylpyrazole (2a) and allyl alcohol.

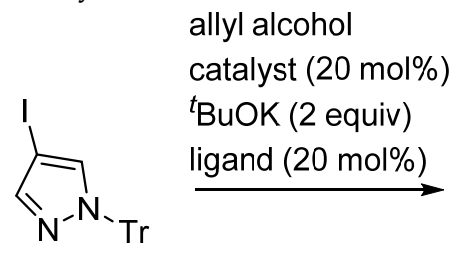

$2 \mathbf{a}$

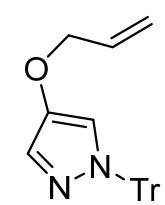

$4 a$

\begin{tabular}{|c|c|c|c|c|c|c|}
\hline Entry & Catalyst & Ligand $^{a}$ & Solvent & Temperature $\left({ }^{\circ} \mathrm{C}\right)$ & Time & 4a Yield, \% \\
\hline $1^{b, c}$ & $\mathrm{Pd}(\mathrm{dba})_{2}$ & L1 & xylene & $160(\mathrm{MW})$ & $30 \mathrm{~min}$ & 0 \\
\hline $2^{c}$ & $\mathrm{CuI}$ & L2 & DMF & 100 & overnight & 0 \\
\hline $3^{c}$ & $\mathrm{CuI}$ & L3 & DMF & 100 & overnight & 0 \\
\hline 4 & $\mathrm{CuI}$ & L3 & allyl alcohol & 100 & overnight & 51 \\
\hline 5 & $\mathrm{CuI}$ & L4 & allyl alcohol & $100(\mathrm{MW})$ & $1 \mathrm{~h}$ & 31 \\
\hline 6 & $\mathrm{CuI}$ & L4 & allyl alcohol & 130 (MW) & $1 \mathrm{~h}$ & 66 \\
\hline 7 & $\mathrm{CuI}$ & L4 & allyl alcohol & 130 (MW) & $30 \mathrm{~min}$ & 24 \\
\hline 8 & $\mathrm{CuI}$ & L4 & allyl alcohol & $160(\mathrm{MW})$ & $1 \mathrm{~h}$ & 16 \\
\hline $9^{d}$ & $\mathrm{CuI}$ & L4 & allyl alcohol & $130(\mathrm{MW})$ & $1 \mathrm{~h}$ & 37 \\
\hline
\end{tabular}

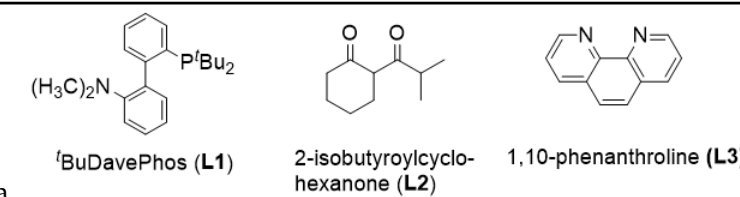
hexanone (L2)

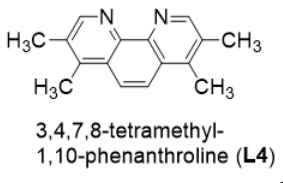

1,10 -phenanthroline $(14)$

b. $10 \mathrm{~mol} \% \operatorname{Pd}(\mathrm{dba})_{2}$ was used, c. 2 equiv of allyl

\subsection{C4-Alkoxylation of 4-iodopyrazole with Alcohols Using CuI-Catalyzed Coupling}

To study the scope and limitations of this transformation, the CuI-catalyzed reactions of iodopyrazoles $2(50 \mathrm{mg})$ with various alcohols $(2.0 \mathrm{~mL}$, excess amount) were carried out under the optimal conditions (Table 1, entry 6). The results are summarized in Table 2. The reactions of 2a with linear short-chain primary alcohols (methanol, ethanol, and $n$-propanol) afforded the corresponding products $4 \mathbf{c}, 4 \mathbf{d}$, and $4 \mathbf{e}$ in moderate yields (61-76\%, entries $1-3)$, while the reaction with a longer-chain primary alcohol ( $n$-butanol) resulted in a lower yield (33\%, entry 4). The reactions of $\mathbf{2 a}$ with branched primary alcohols (isobutyl and isoamyl alcohols) provided $4 \mathbf{i}(45 \%)$ and $4 \mathbf{k}(37 \%)$, respectively (entries 7 and 9 ), but with secondary isopropanol, gave $\mathbf{4 g}$ in only $9 \%$ yield (entry 5 ). The presence of sec- or tert-butyl groups in the alcohol was not compatible with the present reaction conditions (entries 6 and 8), probably due to steric hindrance. In contrast, the reactions with cyclic secondary alcohols did proceed (entries 10,11, and 12), but the respective isolated yields of the coupled products $41,4 \mathrm{~m}$, and $4 \mathrm{n}$ were $59 \%, 18 \%$, and $25 \%$, respectively. In these reactions, $1.0 \mathrm{~mL}$ of cyclic alcohol was used with respect to substrate $2 \mathrm{a}(50 \mathrm{mg})$; the high boiling points (cyclobutanol: $123^{\circ} \mathrm{C} / 733 \mathrm{mmHg}$; cyclopentanol: $139-140^{\circ} \mathrm{C}$; cyclohexanol: $160-161^{\circ} \mathrm{C}$ ) of these materials complicated product isolation by chromatography. Furthermore, when 2 equivalents of the cyclic alcohols and acetonitrile $(2.0 \mathrm{~mL})$ as a co-solvent were used, 
no coupled products could be detected. Although the reaction with benzyl alcohol (bp: $\left.205^{\circ} \mathrm{C}\right)$ was also difficult, the use of benzyl alcohol $(1.0 \mathrm{~mL})$ and toluene as a co-solvent $(1.0 \mathrm{~mL})$ afforded the corresponding product $(40)$ in poor yield $(12 \%$, entry 13$)$. With phenols, no desired coupling products were obtained under various reaction conditions (entries 14 and 15). In the case of p-methoxyphenol (entry 15), a detailed analysis of the reaction mixture revealed a trace amount of 5,5'-dimethoxy-2,2'-biphenyldiol, which has been reported to have radical scavenging or antibacterial activities [32,33]. The initially formed dihydroxybiphenyls [34] might inhibit the attempted $\mathrm{C}-\mathrm{O}$ coupling reaction.

Table 2. CuI-catalyzed coupling reaction between iodopyrazoles and various alcohols.

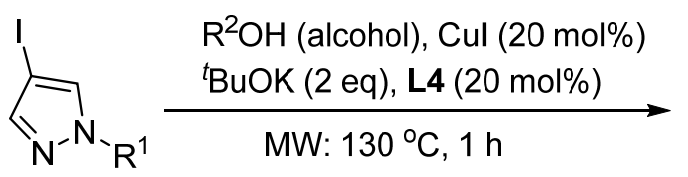

2

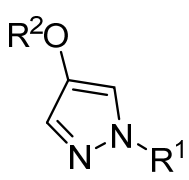

4

\begin{tabular}{|c|c|c|c|c|}
\hline Entry & Substrate & $\mathrm{R}^{2} \mathrm{OH}$ & Product & Yield (\%) \\
\hline 1 & 2a: $R^{1}=T r$ & $\mathrm{R}^{2}=\mathrm{Me}$ & 4c: $\mathrm{R}^{1}=\mathrm{Tr}, \mathrm{R}^{2}=\mathrm{Me}$ & 61 \\
\hline 2 & $2 a$ & $\mathrm{R}^{2}=\mathrm{Et}$ & $4 d: R^{1}=T r, R^{2}=E t$ & 76 \\
\hline 3 & $2 a$ & $\mathrm{R}^{2}=n-\operatorname{Pr}$ & $4 \mathrm{e}: \mathrm{R}^{1}=\mathrm{Tr}, \mathrm{R}^{2}=n-\mathrm{Pr}$ & 64 \\
\hline 4 & $2 a$ & $\mathrm{R}^{2}=n-\mathrm{Bu}$ & 4f: $\mathrm{R}^{1}=\operatorname{Tr}, \mathrm{R}^{2}=n-B u$ & 33 \\
\hline 5 & $2 a$ & $\mathrm{R}^{2}=i \mathrm{Pr}$ & 4g: $\mathrm{R}^{1}=\mathrm{Tr}, \mathrm{R}^{2}=i \operatorname{Pr}$ & 9 \\
\hline 6 & $2 a$ & $\mathrm{R}^{2}=\sec -\mathrm{Bu}$ & 4h: $\mathrm{R}^{1}=\mathrm{Tr}, \mathrm{R}^{2}=\sec -\mathrm{Bu}$ & 0 \\
\hline 7 & $2 a$ & $\mathrm{R}^{2}=i \mathrm{Bu}$ & $4 \mathrm{i}: \mathrm{R}^{1}=\mathrm{Tr}, \mathrm{R}^{2}=i \mathrm{Bu}$ & 45 \\
\hline 8 & $2 a$ & $\mathrm{R}^{2}=$ tert $-\mathrm{Bu}$ & $4 \mathbf{j}: \mathrm{R}^{1}=\mathrm{Tr}, \mathrm{R}^{2}=$ tert $-\mathrm{Bu}$ & 0 \\
\hline 9 & $2 a$ & $\mathrm{R}^{2}=$ isoamyl & 4k: $\mathrm{R}^{1}=\mathrm{Tr}, \mathrm{R}^{2}=$ isoamyl & 37 \\
\hline $10^{\mathrm{a}}$ & $2 a$ & $\mathrm{R}^{2}=$ cyclobutyl & 41: $\mathrm{R}^{1}=\mathrm{Tr}, \mathrm{R}^{2}=$ cyclobutyl & 59 \\
\hline $11^{\mathrm{a}}$ & $2 a$ & $\mathrm{R}^{2}=$ cyclopentyl & $4 \mathrm{~m}: \mathrm{R}^{1}=\mathrm{Tr}, \mathrm{R}^{2}=$ cyclopentyl & 18 \\
\hline $12^{\mathrm{a}}$ & $2 a$ & $\mathrm{R}^{2}=$ cyclohexyl & 4n: $\mathrm{R}^{1}=\mathrm{Tr}, \mathrm{R}^{2}=$ cyclohexyl & 25 \\
\hline $13^{b}$ & $2 a$ & $\mathrm{R}^{2}=\mathrm{Bn}$ & 4o: $\mathrm{R}^{1}=\mathrm{Tr}, \mathrm{R}^{2}=\mathrm{Bn}$ & 12 \\
\hline 14 & $2 a$ & $\mathrm{R}^{2}=\mathrm{Ph}$ & $4 p: \mathrm{R}^{1}=\mathrm{Tr}, \mathrm{R}^{2}=\mathrm{Ph}$ & 0 \\
\hline $15^{c}$ & $2 a$ & $\mathrm{R}^{2}=p-\mathrm{MeOPh}$ & 4q: $\mathrm{R}^{1}=\mathrm{Tr}, \mathrm{R}^{2}=p-\mathrm{MeOPh}$ & 0 \\
\hline $16^{\mathrm{d}}$ & $2 a$ & $\mathrm{R}^{2}=$ allyl & 4a: $R^{1}=\operatorname{Tr}, R^{2}=$ allyl & 66 \\
\hline 17 & 2b: $\mathrm{R}^{1}=1$-propenyl & $\mathrm{R}^{2}=1$-propenyl & 4r: $R^{1}=1$-propenyl, $R^{2}=$ allyl & 65 \\
\hline 18 & $2 \mathrm{c}: \mathrm{R}^{1}=$ allyl & $\mathrm{R}^{2}=$ allyl & $4 s: R^{1}=R^{2}=$ allyl & 58 \\
\hline 19 & 2d: $\mathrm{R}^{1}=3$-butenyl & $\mathrm{R}^{2}=$ allyl & $4 t: R^{1}=3$-butenyl, $R^{2}=$ allyl & 64 \\
\hline $20^{e}$ & $2 a$ & $\mathrm{R}^{2}=\mathrm{OH}$ & $4 \mathbf{u}: \mathrm{R}^{1}=\mathrm{Tr}, \mathrm{R}^{2}=\mathrm{H}$ & 0 \\
\hline 21 & 1 & $\mathrm{R}^{2}=$ allyl & $4 \mathbf{v}: \mathrm{R}^{1}=\mathrm{H}, \mathrm{R}^{2}=$ allyl & 0 \\
\hline
\end{tabular}

a. $1.0 \mathrm{~mL}$ of cyclic alcohol was used, b. $1.0 \mathrm{~mL}$ of $\mathrm{BnOH}$ was used with toluene $(1.0 \mathrm{~mL})$ as co-solvent, c. Trace amount of 5,5'-dimethoxy2,2'-biphenyldiol was isolated, d. Table 1, entry 6, e. 2a was recovered (89\%).

The direct introduction of the allyloxy group at the $\mathrm{C} 4$ position of $\mathrm{N}$-alkenyl-4-iodo$1 H$-pyrazoles $(\mathbf{2} \mathbf{b}, \mathbf{2 c}, \mathbf{2 d})$ by CuI-mediated reaction afforded the expected products $4 \mathbf{r}, \mathbf{4 s}$, and $\mathbf{4 t}$ in moderate yields (entries 17-19). These products were subsequently applied in the synthesis of withasomnine and its analogs (Scheme 2). Neither the $\mathrm{C}-\mathrm{O}$ coupling reaction of $2 \mathbf{a}$ with water nor of $\mathrm{N}$-nonprotected iodopyrazole $\mathbf{1}$ with allyl alcohol was successful (entries 20 and 21).

In preliminary experiments, the $\operatorname{Pd}(\mathrm{dba})_{2}$-catalyzed coupling of $\mathbf{2 a}$ with four types of alcohols (methanol, ethanol, $n$-propanol, and tert-butyl alcohol) under the same conditions as mentioned above was examined; however, these trials did not give the desired coupling products, yielding only hydrogenated $1 \mathrm{H}$-1-tritylpyrazole in $52,63,64$, and $8 \%$ yields, respectively. 

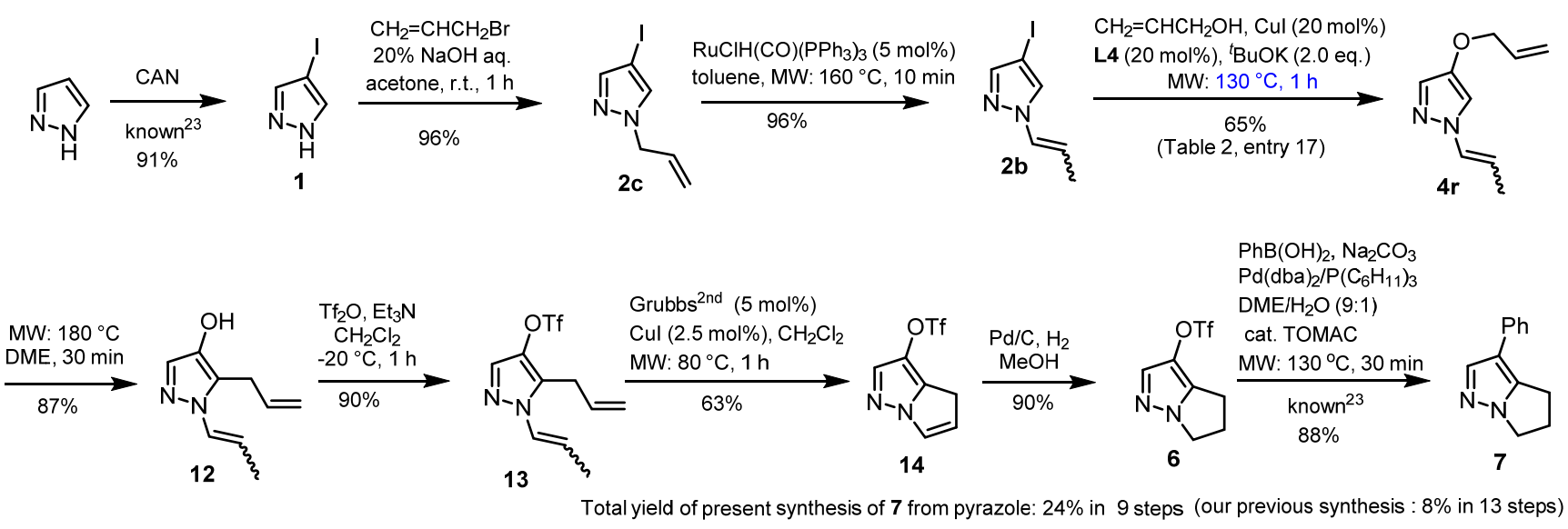

Scheme 2. Application to improved synthesis of withasomnine (7).

\subsection{Application to Improved Synthesis of Withasomnine and Six- and Seven-Membered Cyclic Homologs}

A modified synthesis of withasomnine and its homologs using the products described above was performed to demonstrate the usefulness of the present method. The improved synthesis of withasomnine (7) is summarized in Scheme 2. 4-Iodo- $1 H$-pyrazole (1) was treated with allyl bromide under basic conditions to give $N$-allylated compound $2 \mathrm{c}$ in $97 \%$ yield. The double bond in the $N$-allyl group in $2 \mathrm{c}$ was migrated by treatment with a ruthenium hydride catalyst $\left(\mathrm{RuClH}(\mathrm{CO})\left(\mathrm{PPh}_{3}\right)_{3}\right)$ to give an $\mathrm{E} / \mathrm{Z}$ mixture of $\mathbf{2 b}$ in $96 \%$ yield, which was transformed to $4 \mathbf{r}$ by CuI-catalyzed coupling, as described above (Table 2, entry 17). The Claisen rearrangement of $4 \mathbf{r}$ gave $(E / Z)-12(87 \%)$, which was subsequently $O$-triflated by treatment with trifluoromethanesulfonic anhydride $\left(\mathrm{Tf}_{2} \mathrm{O}\right)$ in the presence of triethylamine at $-20{ }^{\circ} \mathrm{C}$ to yield ring-closing metathesis (RCM) substrate 13 in $90 \%$ yield. Treatment of 13 with Grubbs ${ }^{2 n d}$ catalyst in toluene at $100{ }^{\circ} \mathrm{C}$ under MW irradiation gave the desired RCM product 14 in $0-58 \%$ yields with unsatisfied reproducibility.

Alternatively, CuI-assisted RCM [24,35] of $\mathbf{1 3}$ in $\mathrm{CH}_{2} \mathrm{Cl}_{2}$ under milder conditions using MW-aided heating at $80^{\circ} \mathrm{C}$ for $1 \mathrm{~h}$ successfully afforded pyrrole-[1,2- $\left.b\right]$ pyrazole $14(63 \%)$, which was immediately hydrogenated under a hydrogen gas atmosphere with $\mathrm{Pd} / \mathrm{C}$ in $\mathrm{MeOH}$ to give penultimate product 6 in $90 \%$ yield. As the transformation from 6 to 7 via a Suzuki-Miyaura coupling reaction has already been reported [22,23], the present approach constitutes a formal total synthesis of withasomnine (7). The overall yield of 7 in this case was $24 \%$ over nine steps from commercially available pyrazole, whereas that of our previous method was $8 \%$ in 13 steps. Therefore, the current synthesis realizes a four-step reduction and nearly threefold improvement in overall yield [22,23].

The syntheses of the six-and seven-membered cyclic homologs 11 and 15 are summarized in Scheme 3. The total yield of $\mathbf{1 1}$ was improved by $\sim 1.6$-fold over our former synthesis based on the yields of transformations from 1 to $2 c$ (seen in Scheme 2) and $2 \mathrm{c}$ to $4 \mathbf{s}$ (Table 2, entry 18) [24]. Our synthesis of another withasomnine homolog, 15, previously achieved by Allin via radical cyclization [25,26], began with the transformation of $\mathbf{1}$ to $\mathbf{2 d}$ in $88 \%$ yield. Compound $\mathbf{2 d}$ was $O$-allylated using the present method to $4 \mathbf{t}$, as described previously (Table 2, entry 19$)$. Then, $\mathbf{4 t}$ was rearranged into $\mathbf{1 6}$ (81\% yield) under $\mathrm{MW}$-assisted heating, and subsequent $\mathrm{O}$-triflation afforded 17 (83\% yield). RCM substrate 17 was similarly cyclized to seven-membered intermediate 18 in $72 \%$ yield, which was then subjected to Suzuki-Miyaura coupling with phenylboronic acid to afford 19 in $87 \%$ yield. The synthesis of $\mathbf{1 5}$ was completed in $92 \%$ yield by the Pd-C-catalyzed hydrogenation of 19. An alternative route to 15 comprised the transformation of 1 to $4 \mathrm{~b}$ in $52 \%$ yield via a five-step process, and subsequent $N$-butenylation to give the common intermediate $4 \mathbf{t}$ in $69 \%$ yield. Therefore, the present route to 15 using the CuI-catalyzed coupling achieved a 1.6-fold increase in overall yield compared to the prior procedure. 


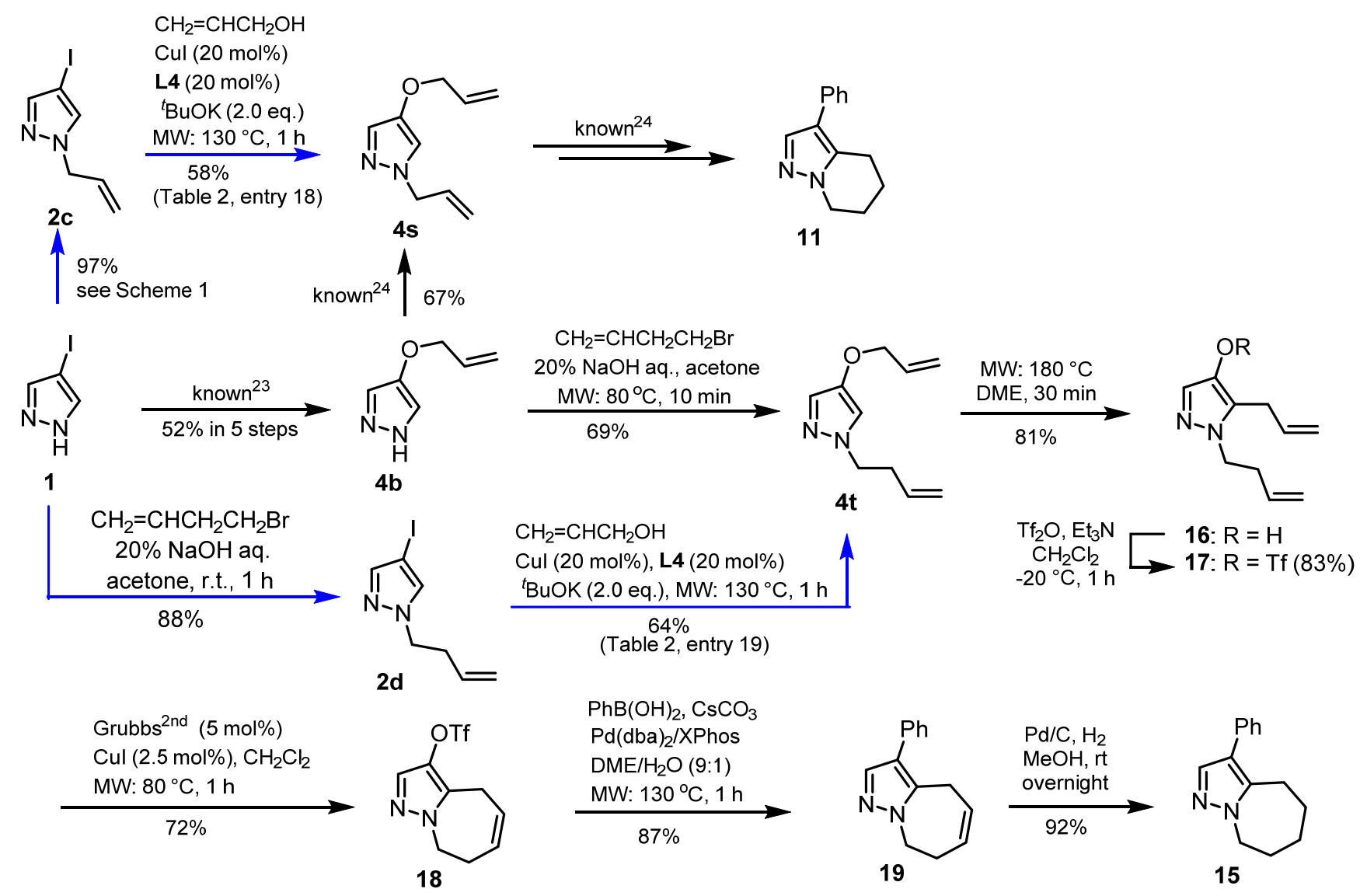

Scheme 3. Synthesis of withasomnine six- and seven-membered cyclic homologs $\mathbf{1 1}$ and 15.

\section{Conclusions}

In this study, a range of 4-alkoxy- $1 H$-pyrazoles was synthesized using the CuIcatalyzed coupling reaction of 4-iodopyrazoles with an excess amount of alcohol. Improved syntheses of withasomnine and its homologs were achieved using the products obtained with the present method. The current withasomnine synthetic route was reduced by four steps with a threefold-improvement in the overall yield compared to our previous report $[22,23]$. However, reducing the amounts of catalysts, ligands, and alcohols will be required to increase the practicality of this reaction in the future.

\section{Materials and Methods}

\subsection{General Information}

NMR spectra were recorded at $27^{\circ} \mathrm{C}$ on Agilent 400- and 600-MR-DD2 spectrometers (Agilent Tech., Inc., Santa Clara, CA, USA) in $\mathrm{CDCl}_{3}$ with tetramethylsilane (TMS) as an internal standard. HRMS was performed using a JEOL JMS-700 (2) mass spectrometer (JEOL, Tokyo, Japan). Melting points were determined using a Yanagimoto micromelting point apparatus (Yamagimoto, Kyoto, Japan) and were uncorrected. Liquid column chromatography was conducted using silica gel (Fuji Silysia FL-60D). Analytical TLC was performed on precoated Merck glass plates (silica gel $60 \mathrm{~F}_{254}$ ), and compounds were detected by dipping the plates in an ethanol solution of phosphomolybdic acid, followed by heating. All microwave-aided reactions were performed using a Biotage Initiator ${ }^{\circledR}$ (Biotage, Uppsala, Sweden). Allyl alcohol, $n$-propanol, isobutyl alcohol, cyclobutanol, cyclopentanol, cyclohexanol, Phenol, p-methoxyphenol, allylbromide, and $\mathrm{Pd}(\mathrm{dba})_{2}$, were purchased from Tokyo Chemical Industry (TCI) Co. (Tokyo, Japan). t-BuOK, CuI, 1,10-phenanthroline (L3), 3,4,7,8tetramethyl-1,10-phenanthroline (L4), isopropanol, tert-butanol, isoamyl alcohol, toluene, cesium acetate, trifluoromethanesulfonic anhydride, and triethylamine were purchased 
from Nacalai Tesque, Inc. (Kyoto, Japan). Dry xylene, dry DMF, $n$-butanol, sec-butanol, and 1,2-dimethoxyethane, were purchased from FUJIFILM Wako Pure Chemical Co. (Osaka, Japan). tBuDavePhos (L1), 2-isobutyrylcyclohexanone (L2), $\mathrm{RuClH}(\mathrm{CO})\left(\mathrm{PPh}_{3}\right)_{3}, \mathrm{Grubbs}^{2 \mathrm{nd}}$, and XPhos were purchased from Sigma-Aldrich Co. LLC (St. Louis, MO, USA).

${ }^{1} \mathrm{H}$ - and ${ }^{13} \mathrm{C}-\mathrm{NMR}$ spectra of all new compounds with compound $\mathbf{1 5}$ are provided in Supplementary Materials as Figures S1-S48.

\subsection{CuI-Catalyzed Coupling Reactions of 4-iodo-1H-1-tritylpyrazole with Alcohols (Tables 1 and 2)}

General procedure (Table 1, entry 6): To a solution of $\mathbf{2 a}(50.0 \mathrm{mg}, 0.12 \mathrm{mmol})$ in allyl alcohol (2.0 mL, $29 \mathrm{mmol})$ in a microwave vial $(0.5-2.0 \mathrm{~mL})$ were added 3,4,7,8-tetramethyl1,10-phenanthroline (5.8 mg, $0.026 \mathrm{mmol}, 20 \mathrm{~mol} \%$ ), CuI (4.4 mg, $0.023 \mathrm{mmol}, 20 \mathrm{~mol} \%$ ), and ${ }^{t} \mathrm{BuOK}(28.8 \mathrm{mg}, 0.26 \mathrm{mmol}, 2.0$ equiv). The mixture was stirred to make a solution, sealed, and heated at $130{ }^{\circ} \mathrm{C}$ for $1 \mathrm{~h}$ under MW irradiation. The cooled mixture was checked by TLC (hexane/AcOEt = 8:1), quenched by the addition of saturated (sat.) aqueous (aq.) $\mathrm{NH}_{4} \mathrm{Cl}(1 \mathrm{~mL})$, and extracted with dichloromethane $\left(\mathrm{CH}_{2} \mathrm{Cl}_{2} ; 1.0 \mathrm{~mL} \times 3\right)$. The combined organic layers were dried over $\mathrm{MgSO}_{4}$, filtered, and evaporated to give a crude residue that was purified by silica gel column chromatography (eluent:hexane/AcOEt $=20: 1$ ) to afford previously reported $4 \mathrm{a}(27.9 \mathrm{mg}, 66 \%)$.

4a: known [22,23]

4-Methoxy-1H-1-tritylpyrazole (4c): Colorless needles $\left(\mathrm{CH}_{2} \mathrm{Cl}_{2}\right) ; \mathrm{mp} 149-152{ }^{\circ} \mathrm{C} ;{ }^{1} \mathrm{H}$ NMR $\left(400 \mathrm{MHz}, \mathrm{CDCl}_{3}\right): \delta 7.40(1 \mathrm{H}, \mathrm{s}$, pyrazole-H), 7.32-7.28 $(9 \mathrm{H}, \mathrm{m}, \mathrm{Tr}-\mathrm{H}), 7.18-7.14(6 \mathrm{H}$, $\mathrm{m}, \mathrm{Tr}-\mathrm{H}), 7.01\left(1 \mathrm{H}, \mathrm{s}\right.$, pyrazole-H), $3.68(3 \mathrm{H}, \mathrm{s}, 4-\mathrm{OMe}) ;{ }^{13} \mathrm{C}-\mathrm{NMR}\left(100 \mathrm{MHz}, \mathrm{CDCl}_{3}\right): \delta$ 145.7, 143.2, 130.1, 127.6, 127.4, 127.2, 117.2, 78.6, 58.7; HREIMS $m$ / $z$ calculated (calcd) for $\mathrm{C}_{23} \mathrm{H}_{2} \mathrm{ON}_{2} \mathrm{O}\left(\mathrm{M}^{+}\right) 340.1575$, found 340.1577 .

4-Ethoxy-1H-1-tritylpyrazole (4d): Colorless needles $\left(\mathrm{CH}_{2} \mathrm{Cl}_{2}\right) ; \mathrm{mp} 141-144{ }^{\circ} \mathrm{C} ;{ }^{1} \mathrm{H}$ NMR $\left(400 \mathrm{MHz}, \mathrm{CDCl}_{3}\right): \delta 7.40(1 \mathrm{H}, \mathrm{s}$, pyrazole-H), $7.01(1 \mathrm{H}, \mathrm{s}$, pyrazole-H), 7.31-7.28 $(9 \mathrm{H}$, m, Tr-H), 7.18-7.14 (6H, m, Tr-H), $3.87\left(2 \mathrm{H}, \mathrm{q} J=6.6 \mathrm{~Hz},-\mathrm{OCH}_{2} \mathrm{CH}_{3}\right), 1.32(3 \mathrm{H}, \mathrm{t}, \mathrm{J}=6.6 \mathrm{~Hz}$, $\left.\mathrm{CH}_{2} \mathrm{CH}_{3}\right) ;{ }^{13} \mathrm{C}-\mathrm{NMR}\left(100 \mathrm{MHz}, \mathrm{CDCl}_{3}\right): \delta 144.4,143.2,130.1,127.8,127.6,127.6,117.9,78.6$, 67.1, 14.9; HREIMS $m / z$ calcd for $\mathrm{C}_{24} \mathrm{H}_{22} \mathrm{~N}_{2} \mathrm{O}\left(\mathrm{M}^{+}\right)$354.1732, found 354.1735.

4-n-Propyloxy-1H-1-tritylpyrazole (4e): Colorless needles $\left(\mathrm{CH}_{2} \mathrm{Cl}_{2}\right) ; \mathrm{mp} 118-120^{\circ} \mathrm{C}$; ${ }^{1} \mathrm{H}-\mathrm{NMR}\left(400 \mathrm{MHz}, \mathrm{CDCl}_{3}\right): \delta, 7.40(1 \mathrm{H}, \mathrm{s}$, pyrazole-H), 7.30-7.27 $(9 \mathrm{H}, \mathrm{m}, \mathrm{Tr}-\mathrm{H}), 7.18-7.14$ $(6 \mathrm{H}, \mathrm{m}, \mathrm{Tr}-\mathrm{H}), 7.01\left(1 \mathrm{H}, \mathrm{s}\right.$, pyrazole-H), $3.76\left(2 \mathrm{H}, \mathrm{t}, \mathrm{J}=6.6 \mathrm{~Hz},-\mathrm{OCH}_{2} \mathrm{CH}_{2}-\right), 1.72(2 \mathrm{H}, \mathrm{qt}$, $\left.J=7.4,6.6 \mathrm{~Hz},-\mathrm{CH}_{2} \mathrm{CH}_{2} \mathrm{CH}_{3}\right), 0.97\left(3 \mathrm{H}, \mathrm{t}, J=7.4 \mathrm{~Hz},-\mathrm{CH}_{2} \mathrm{CH}_{3}\right) ;{ }^{13} \mathrm{C}-\mathrm{NMR}(100 \mathrm{MHz}$, $\left.\mathrm{CDCl}_{3}\right): \delta 144.6,143.2,130.1,127.8,127.61,127.59,117.7,78.5,73.1,22.7,10.4 ; \mathrm{HREIMS} \mathrm{m} / z$ calcd for $\mathrm{C}_{25} \mathrm{H}_{24} \mathrm{~N}_{2} \mathrm{O}\left(\mathrm{M}^{+}\right) 368.1889$, found 368.1889.

4-n-Butoxy-1H-1-tritylpyrazole (4f): Colorless needles $\left(\mathrm{CH}_{2} \mathrm{Cl}_{2}\right) ; \operatorname{mp~} 127-130{ }^{\circ} \mathrm{C} ;{ }^{1} \mathrm{H}$ NMR (400 MHz, CDCl $): \delta 7.40(1 \mathrm{H}, \mathrm{s}$, pyrazole-H), 7.31-7.28 $(9 \mathrm{H}, \mathrm{m}, \mathrm{Tr}-\mathrm{H}), 7.18-7.14(6 \mathrm{H}$, $\mathrm{m}, \mathrm{Tr}-\mathrm{H}), 7.01\left(1 \mathrm{H}, \mathrm{s}\right.$, pyrazole-H), $3.80\left(2 \mathrm{H}, \mathrm{q} J=6.6 \mathrm{~Hz},-\mathrm{OCH}_{2} \mathrm{CH}_{2}-\right), 1.68(2 \mathrm{H}$, quint, $\left.J=6.7 \mathrm{~Hz},-\mathrm{CH}_{2} \mathrm{CH}_{2} \mathrm{CH}_{2}-\right), 1.42\left(2 \mathrm{H}\right.$, sext, $\left.J=6.6 \mathrm{~Hz},-\mathrm{CH}_{2} \mathrm{CH}_{2} \mathrm{CH}_{3}\right), 0.93(3 \mathrm{H}, \mathrm{t}, J=6.7 \mathrm{~Hz}$, $\left.-\mathrm{CH}_{2} \mathrm{CH}_{3}\right) ;{ }^{13} \mathrm{C}-\mathrm{NMR}\left(100 \mathrm{MHz}, \mathrm{CDCl}_{3}\right): \delta 144.7,143.2,130.1,127.8,127.6,127.6,117.7,78.5$, 71.3, 31.4, 19.1, 13.9; HREIMS $m / z$ calcd for $\mathrm{C}_{26} \mathrm{H}_{26} \mathrm{~N}_{2} \mathrm{O}\left(\mathrm{M}^{+}\right)$382.2035, found 382.2040.

4-Isopropyloxy-1H-1-tritylpyrazole (4g): White powder; mp 91-93 ${ }^{\circ} \mathrm{C} ;{ }^{1} \mathrm{H}-\mathrm{NMR}$ $\left(400 \mathrm{MHz}, \mathrm{CDCl}_{3}\right): \delta 7.39(1 \mathrm{H}, \mathrm{d}, J=1.0 \mathrm{~Hz}$, pyrazole-H), 7.31-7.28 $(9 \mathrm{H}, \mathrm{m}, \mathrm{Tr}-\mathrm{H}), 7.18-7.14$ $(6 \mathrm{H}, \mathrm{m}, \mathrm{Tr}-\mathrm{H}), 7.02\left(1 \mathrm{H}, \mathrm{d}, J=0.7 \mathrm{~Hz}\right.$, pyrazole-H), $4.12\left(1 \mathrm{H}\right.$, sept, $\left.J=6.1 \mathrm{~Hz},-\mathrm{OCH}\left(\mathrm{CH}_{3}\right)_{2}\right)$, $1.26\left(6 \mathrm{H}, \mathrm{d}, \mathrm{J}=6.1 \mathrm{~Hz},-\mathrm{CH}\left(\mathrm{CH}_{3}\right)_{2}\right) ;{ }^{13} \mathrm{C}-\mathrm{NMR}\left(100 \mathrm{MHz}, \mathrm{CDCl}_{3}\right): \delta 143.2,142.7,130.1$, $129.2,127.6,119.8,78.6,74.0,21.9 ;$ HREIMS $m / z$ calcd for $\mathrm{C}_{25} \mathrm{H}_{24} \mathrm{~N}_{2} \mathrm{O}\left(\mathrm{M}^{+}\right) 368.1889$, found 368.1885.

4-Isobutoxy-1H-1-tritylpyrazole (4i): White powder; mp $95-98{ }^{\circ} \mathrm{C} ;{ }^{1} \mathrm{H}-\mathrm{NMR}(400 \mathrm{MHz}$, $\left.\mathrm{CDCl}_{3}\right): \delta 7.40(1 \mathrm{H}, \mathrm{s}$, pyrazole-H), 7.30-7.28 $(9 \mathrm{H}, \mathrm{m}, \mathrm{Tr}-\mathrm{H}), 7.18-7.14(6 \mathrm{H}, \mathrm{m}, \mathrm{Tr}-\mathrm{H}), 7.02$ $\left(1 \mathrm{H}, \mathrm{s}\right.$, pyrazole-H), $3.56\left(2 \mathrm{H}, \mathrm{d}, J=6.7 \mathrm{~Hz},-\mathrm{OCH}_{2} \mathrm{CH}-\right), 1.99(1 \mathrm{H}$, nonet, $J=6.7 \mathrm{~Hz},-$ $\left.\mathrm{CH}_{2} \mathrm{CH}\left(\mathrm{CH}_{3}\right)_{2}\right), 0.96\left(6 \mathrm{H}, \mathrm{d}, \mathrm{J}=6.7 \mathrm{~Hz},-\mathrm{CH}\left(\mathrm{CH}_{3}\right)_{2}\right) ;{ }^{13} \mathrm{C}-\mathrm{NMR}\left(100 \mathrm{MHz}, \mathrm{CDCl}_{3}\right): \delta 144.8$, $143.2,130.1,127.8,127.65,127.61,127.58,117.6,78.5,78.0,28.4,19.1$; HREIMS $m / z$ calcd for $\mathrm{C}_{26} \mathrm{H}_{26} \mathrm{~N}_{2} \mathrm{O}\left(\mathrm{M}^{+}\right)$382.2035, found 382.2040. 
4-Isoamyloxy-1H-1-tritylpyrazole (4k): White powder; $\mathrm{mp} 77-75^{\circ} \mathrm{C} ;{ }^{1} \mathrm{H}-\mathrm{NMR}(400 \mathrm{MHz}$, $\left.\mathrm{CDCl}_{3}\right): \delta 7.40(1 \mathrm{H}, \mathrm{s}$, pyrazole-H), 7.31-7.29 $(9 \mathrm{H}, \mathrm{m}, \mathrm{Tr}-\mathrm{H}), 7.18-7.14(6 \mathrm{H}, \mathrm{m}, \mathrm{Tr}-\mathrm{H}), 7.01$ $\left(1 \mathrm{H}, \mathrm{s}\right.$, pyrazole-H), $3.83\left(2 \mathrm{H}, \mathrm{q}, J=6.6 \mathrm{~Hz},-\mathrm{OCH}_{2} \mathrm{CH}_{2}-\right), 1.76(1 \mathrm{H}$, nonet, $J=6.7 \mathrm{~Hz}$, $\left.-\mathrm{CH}_{2} \mathrm{CH}\left(\mathrm{CH}_{3}\right)_{2}\right), 1.58\left(2 \mathrm{H}, \mathrm{q}, J=6.7 \mathrm{~Hz},-\mathrm{CH}_{2} \mathrm{CH}_{2} \mathrm{CH}-\right), 0.92\left(6 \mathrm{H}, \mathrm{d}, J=6.7 \mathrm{~Hz},-\mathrm{CH}\left(\mathrm{CH}_{3}\right)_{2}\right)$; ${ }^{13} \mathrm{C}-\mathrm{NMR}\left(100 \mathrm{MHz} \mathrm{CDCl}_{3}\right): \delta 22.6,24.8,38.1,70.0,78.6,117.8,127.6,127.7,127.8,130.1$, 143.2, 144.6; HREIMS $m / z$ calcd for $\mathrm{C}_{26} \mathrm{H}_{26} \mathrm{~N}_{2} \mathrm{O}\left(\mathrm{M}^{+}\right)$396.2201, found 396.2201.

4-Cyclobutyloxy-1H-1-tritylpyrazole (41): White powder; mp $119-121{ }^{\circ} \mathrm{C} ;{ }^{1} \mathrm{H}-\mathrm{NMR}$ $\left(400 \mathrm{MHz}, \mathrm{CDCl}_{3}\right): \delta 7.34(1 \mathrm{H}$, br s, pyrazole-H), 7.31-7.28 $(9 \mathrm{H}, \mathrm{m}, \mathrm{Tr}-\mathrm{H}), 7.17-7.12(6 \mathrm{H}, \mathrm{m}$, $\left.\mathrm{Tr}-\mathrm{H}), 6.95\left(1 \mathrm{H} \text {, br s, pyrazole-H), 4.40-4.33 (1H, m, -OCH( } \mathrm{CH}_{2}\right)_{2}-\right), 2.31-2.33(2 \mathrm{H}, \mathrm{m}), 2.13-$ $2.03(2 \mathrm{H}, \mathrm{m}), 1.81-1.73(1 \mathrm{H}, \mathrm{m}), 1.62-1.52(1 \mathrm{H}, \mathrm{m}) ;{ }^{13} \mathrm{C}-\mathrm{NMR}\left(100 \mathrm{MHz}, \mathrm{CDCl}_{3}\right): \delta 143.2$, $142.4,130.1,128.3,127.6,118.6,78.6,74.4,30.3,12.6$; HREIMS $m / z$ calcd for $\mathrm{C}_{26} \mathrm{H}_{24} \mathrm{~N}_{2} \mathrm{O}$ $\left(\mathrm{M}^{+}\right)$380.1889, found 380.1890 .

4-Cyclopentyloxy-1H-1-tritylpyrazole (4m): White powder; mp $104-106{ }^{\circ} \mathrm{C} ;{ }^{1} \mathrm{H}-\mathrm{NMR}$ $\left(400 \mathrm{MHz}, \mathrm{CDCl}_{3}\right): \delta 7.37(1 \mathrm{H}, \mathrm{d}, J=0.6 \mathrm{~Hz}$, pyrazole-H), 7.37-7.28 $(9 \mathrm{H}, \mathrm{m}, \mathrm{Tr}-\mathrm{H}), 7.17-7.12$ $(6 \mathrm{H}, \mathrm{m}, \mathrm{Tr}-\mathrm{H}), 6.98\left(1 \mathrm{H}, \mathrm{d}, J=0.8 \mathrm{~Hz}\right.$, pyrazole-H), 4.44-4.42 $\left(1 \mathrm{H}, \mathrm{m},-\mathrm{OCH}\left(\mathrm{CH}_{2}\right)_{2}-\right), 1.82-$ $1.74(4 \mathrm{H}, \mathrm{m}), 1.72-1.56(2 \mathrm{H}, \mathrm{m}) ;{ }^{13} \mathrm{C}-\mathrm{NMR}\left(100 \mathrm{MHz}, \mathrm{CDCl}_{3}\right): \delta 143.2,130.1,128.7,127.8$, 127.7, 127.6, 119.0, 83.1, 78.5, 32.6, 23.8; HREIMS $m / z$ calcd for $\mathrm{C}_{27} \mathrm{H}_{26} \mathrm{~N}_{2} \mathrm{O}\left(\mathrm{M}^{+}\right)$394.2045, found 394.2043 .

4-Cyclohexyloxy-1H-1-tritylpyrazole (4n): White powder; mp $132-135{ }^{\circ} \mathrm{C} ;{ }^{1} \mathrm{H}-\mathrm{NMR}$ $\left(400 \mathrm{MHz} \mathrm{CDCl}_{3}\right): \delta 7.39(1 \mathrm{H}, \mathrm{s}$, pyrazole-H), 7.31-7.28 $(9 \mathrm{H}, \mathrm{m}, \mathrm{Tr}-\mathrm{H}), 7.17-7.12(6 \mathrm{H}, \mathrm{m}$, $\mathrm{Tr}-\mathrm{H}), 7.03\left(1 \mathrm{H}, \mathrm{s}\right.$, pyrazole-H), 3.78-3.83 (1H, m, -OCH $\left.\left(\mathrm{CH}_{2}\right)_{2}-\right), 1.95-1.92(2 \mathrm{H}, \mathrm{m}), 1.57-1.51$ $(2 \mathrm{H}, \mathrm{m}), 1.48-1.38(2 \mathrm{H}, \mathrm{m}) 1.32-1.23(2 \mathrm{H}, \mathrm{m}) ;{ }^{13} \mathrm{C}-\mathrm{NMR}\left(100 \mathrm{MHz}, \mathrm{CDCl}_{3}\right): \delta 143.2,142.5$, $130.1,129.5,127.6,120.1,79.6,78.6,31.8,25.6,23.6$; HREIMS $m / z$ calcd for $\mathrm{C}_{28} \mathrm{H}_{28} \mathrm{~N}_{2} \mathrm{O}\left(\mathrm{M}^{+}\right)$ 408.2202, found 408.2201 .

4-Benzyloxy-1H-1-tritylpyrazole (4o): White powder; mp $154-156{ }^{\circ} \mathrm{C} ;{ }^{1} \mathrm{H}-\mathrm{NMR}(400 \mathrm{MHz}$, $\left.\mathrm{CDCl}_{3}\right): \delta 7.44(1 \mathrm{H}, \mathrm{s}$, pyrazole-H), 7.35-7.28 (14H, m, Tr-H, Ph-H), 7.15-7.12 (6H, m, Tr-H, $\mathrm{Ph}-\mathrm{H}), 7.02\left(1 \mathrm{H}, \mathrm{s}\right.$, pyrazole-H), $4.86\left(2 \mathrm{H}\right.$, br s, $\left.-\mathrm{OCH}_{2} \mathrm{Ph}\right) ;{ }^{13} \mathrm{C}-\mathrm{NMR}\left(100 \mathrm{MHz}, \mathrm{CDCl}_{3}\right): \delta$ $144.1,143.1,136.7,130.1,128.5,128.0,127.8,127.6,118.7,78.6,73.7$; HREIMS $m / z$ calcd for $\mathrm{C}_{29} \mathrm{H}_{24} \mathrm{~N}_{2} \mathrm{O}\left(\mathrm{M}^{+}\right) 416.1889$, found 416.1889 .

(E/Z)-4-(Allyloxy)-1-(prop-1-en-1-yl)-1H-pyrazole (4r): Colorless oil; ${ }^{1} \mathrm{H}-\mathrm{NMR}(400 \mathrm{MHz}$, $\left.\mathrm{CDCl}_{3}\right): \delta 7.38(0.1 \mathrm{H}, \mathrm{s}$, pyrazole-H), $7.32(0.7 \mathrm{H}$, br s, pyrazole- $\mathrm{H}), 7.28(0.3 \mathrm{H}, \mathrm{d}, J=0.6$ $\mathrm{Hz}$, pyrazole-H), $7.23(0.7 \mathrm{H}, \mathrm{d}, J=0.8 \mathrm{~Hz}$, pyrazole-H), $6.74(0.7 \mathrm{H}, \mathrm{dq}, J=14.2,0.7 \mathrm{~Hz}$, $\left.(E)-\mathrm{NCH}=\mathrm{CHCH}_{3}\right), 6.68\left(0.3 \mathrm{H}, \mathrm{dq}, J=9.4,0.7 \mathrm{~Hz},(\mathrm{Z})-\mathrm{NCH}=\mathrm{CHCH}_{3}\right), 6.08-5.97(1 \mathrm{H}$, $\left.\mathrm{m},-\mathrm{OCH}_{2} \mathrm{CH}=\mathrm{CH}_{2}\right), 5.85\left(0.7 \mathrm{H}, \mathrm{dq}, J=14.2,7.0 \mathrm{~Hz},(E)-\mathrm{NCH}=\mathrm{CHCH}_{3}\right), 5.40(1 \mathrm{H}, \mathrm{br} \mathrm{d}$, $J=17.2 \mathrm{~Hz},-\mathrm{CH}=\mathrm{CHH}), 5.29(1 \mathrm{H}, \mathrm{br} \mathrm{d}, J=9.4 \mathrm{~Hz},-\mathrm{CH}=\mathrm{CH}), 5.26(0.3 \mathrm{H}, \mathrm{dq}, J=9.4,7.9$, (Z)-NCH=CHCH 3$), 4.44\left(0.6 \mathrm{H}, \mathrm{dt}, J=5.5,1.2 \mathrm{~Hz},-\mathrm{OCH}_{2} \mathrm{CH}=\mathrm{CH}_{2}\right), 4.42(1.4 \mathrm{H}, \mathrm{dt}, J=5.5$, $\left.1.5 \mathrm{~Hz},-\mathrm{OCH}_{2} \mathrm{CH}=\mathrm{CH}_{2}\right), 1.95\left(0.9 \mathrm{H}, \mathrm{dd}, J=7.4,1.8 \mathrm{~Hz},(\mathrm{Z})-\mathrm{NCH}=\mathrm{CHCH}_{3}\right), 1.81(2.1 \mathrm{H}$, $\left.\mathrm{dd}, J=7.1,0.6 \mathrm{~Hz},(E)-\mathrm{NCH}=\mathrm{CHCH}_{3}\right) ;{ }^{13} \mathrm{C}-\mathrm{NMR}\left(100 \mathrm{MHz}, \mathrm{CDCl}_{3}\right): \delta 146.0,133.1,133.0$, $128.8,128.72,127.67,127.65,118.1,115.0,111.8,111.1,72.55,72.47,14.7,12.8$ (3 carbons are overlapped); HREIMS $m / z$ calcd for $\mathrm{C}_{9} \mathrm{H}_{12} \mathrm{~N}_{2} \mathrm{O}\left(\mathrm{M}^{+}\right)$164.0950, found 164.0949.

4s: known [24]

4-Allyloxy-1-(3-buten-1-yl)pyrazole (4t): Colorless oil; ${ }^{1} \mathrm{H}-\mathrm{NMR}$ (400 MHz, $\mathrm{CDCl}_{3}$ ): $\delta 7.24(1 \mathrm{H}, \mathrm{d}, J=1.2 \mathrm{~Hz}$, pyrazole-H), $7.08(1 \mathrm{H}, \mathrm{d}, J=1.0 \mathrm{~Hz}$, pyrazole-H), $6.01(1 \mathrm{H}$, ddt, $\left.J=17.2,10.5,5.5 \mathrm{~Hz},-\mathrm{OCH}_{2} \mathrm{CH}=\mathrm{CH}_{2}\right), 5.74\left(1 \mathrm{H}, \mathrm{ddt}, J=17.2,10.4,6.8 \mathrm{~Hz},-\mathrm{CH}_{2} \mathrm{CH}=\mathrm{CH}_{2}\right)$, $5.38\left(1 \mathrm{H}, \mathrm{dq}, J=17.2,1.6 \mathrm{~Hz},-\mathrm{CH}_{2} \mathrm{CH}=\mathrm{CHH}\right), 5.28\left(1 \mathrm{H}, \mathrm{dq}, J=10.4,1.4 \mathrm{~Hz},-\mathrm{CH}_{2} \mathrm{CH}=\mathrm{CH}\right)$, 5.04-5.10 (2H, overlapped, $2 \times-\mathrm{CH}=\mathrm{CHH}), 4.40\left(2 \mathrm{H}, \mathrm{dt}, \mathrm{J}=5.4,1.5 \mathrm{~Hz},-\mathrm{OCH}_{2} \mathrm{CH}=\right), 4.07$ $\left(2 \mathrm{H}, \mathrm{t}, J=7.1 \mathrm{~Hz}, \mathrm{NCH}_{2} \mathrm{CH}_{2}-\right), 2.57\left(2 \mathrm{H}, \mathrm{qt}, \mathrm{J}=7.0,1.2 \mathrm{~Hz},-\mathrm{CH}_{2} \mathrm{CH}_{2} \mathrm{CH}=\mathrm{CH}_{2}\right)$; ${ }^{13} \mathrm{C}-\mathrm{NMR}$ $\left(100 \mathrm{MHz}_{\mathrm{CDCl}}\right.$ ): $\mathrm{CD}$ 144.9, 134.1, 133.3, 127.0, 117.8, 117.4, 115.0, 72.5, 52.1, 34.5; HREIMS $m / z$ calcd for $\mathrm{C}_{10} \mathrm{H}_{14} \mathrm{~N}_{2} \mathrm{O}\left(\mathrm{M}^{+}\right)$178.1106, Found 178.1105 .

\subsection{Modified Synthesis of Withasomnine, (Scheme 2)}

4.3.1. Synthesis of 1-allyl-4-iodo-1H-pyrazole (2c)

To a solution of 4-iodopyrazole $1(500.0 \mathrm{mg}, 2.6 \mathrm{mmol})$ in acetone $(5 \mathrm{~mL}), 20 \% \mathrm{NaOH}$ aq. ( $0.5 \mathrm{~mL}, 1.5$ equiv) was added with stirring followed by allyl bromide $(0.2 \mathrm{~mL}, 3.9 \mathrm{mmol}$, 
1.5 equiv). The reaction mixture was stirred at room temperature for $1 \mathrm{~h}$. After checking by TLC (hexane/ $\mathrm{AcOEt}=2: 1$ ), sat. aq. $\mathrm{NH}_{4} \mathrm{Cl}(5 \mathrm{~mL})$ was added to the reaction mixture to quench the reaction. The mixture was extracted with $\mathrm{CH}_{2} \mathrm{Cl}_{2}(10 \mathrm{~mL} \times 3)$ and the combined organic layers were washed with brine $(5 \mathrm{~mL} \times 2)$, dried over $\mathrm{MgSO}_{4}$, and filtered. The solvent was removed under reduced pressure to give a crude residue that was purified by silica gel column chromatography (eluent:hexane/ AcOEt $=2: 1)$ to afford $2 \mathrm{c}(566.2 \mathrm{mg}$, 96\%): colorless oil; ${ }^{1} \mathrm{H}-\mathrm{NMR}\left(400 \mathrm{MHz}, \mathrm{CDCl}_{3}\right): \delta 7.53(1 \mathrm{H}, \mathrm{s}$, pyrazole- $\mathrm{H}), 7.45(1 \mathrm{H}, \mathrm{s}$, pyrazole- $\mathrm{H}), 6.00\left(1 \mathrm{H}, \mathrm{ddt}, J=17.1,10.2,6.1 \mathrm{~Hz},-\mathrm{NHCH}_{2} \mathrm{CH}=\mathrm{CH}_{2}\right), 5.30(1 \mathrm{H}, \mathrm{dq}, J=10.2$, $\left.1.2 \mathrm{~Hz},-\mathrm{CH}_{2} \mathrm{CH}=\mathrm{CHH}\right), 5.26\left(1 \mathrm{H}, \mathrm{dq}, J=17.1,1.4 \mathrm{~Hz},-\mathrm{CH}_{2} \mathrm{CH}=\mathrm{CHH}\right), 4.75(2 \mathrm{H}, \mathrm{dt}, J=6.3$, $\left.1.4 \mathrm{~Hz},-\mathrm{NCH}_{2} \mathrm{CH}=\mathrm{CH}_{2}\right) ;{ }^{13} \mathrm{C}-\mathrm{NMR}\left(100 \mathrm{MHz}, \mathrm{CDCl}_{3}\right) \delta$ 144.4, 133.4, 132.2, 119.3, 56.2, 55.1; HREIMS $m / z$ calcd for $\mathrm{C}_{6} \mathrm{H}_{7} \mathrm{~N}_{2} \mathrm{I}\left(\mathrm{M}^{+}\right)$233.9654, found 233.9653 .

\subsubsection{Synthesis of (E/Z)-4-iodo-1-(prop-1-en-1-yl)-1H-pyrazole (2b)}

To a MW vial containing a solution of $2 \mathrm{c}(571.5 \mathrm{mg}, 2.4 \mathrm{mmol})$ in toluene $(2 \mathrm{~mL})$ was added the ruthenium hydride catalyst, $\mathrm{RuClH}(\mathrm{CO})\left(\mathrm{PPh}_{3}\right)_{3}(116.3 \mathrm{mg}, 0.12 \mathrm{mmol}$, $5 \mathrm{~mol} \%$ ). The reaction mixture in the sealed vial was heated at $160{ }^{\circ} \mathrm{C}$ for $10 \mathrm{~min}$ under MW irradiation. After removal of the solvent from the mixture, the residue was purified by silica gel column chromatography (eluent:hexane $/ \mathrm{AcOEt}=10: 1)$ to afford $\mathbf{2 b}(546.6 \mathrm{mg}$, $96 \%)$ as a colorless oil: ${ }^{1} \mathrm{H}-\mathrm{NMR}\left(400 \mathrm{MHz}, \mathrm{CDCl}_{3}\right): \delta 7.62(0.2 \mathrm{H}, \mathrm{s}$, pyrazole- $\mathrm{H}), 7.61(0.2 \mathrm{H}$, $\mathrm{s}$, pyrazole- $\mathrm{H}), 7.58(0.8 \mathrm{H}, \mathrm{s}$, pyrazole- $\mathrm{H}), 7.55(0.8 \mathrm{H}, \mathrm{s}$, pyrazole- $\mathrm{H}), 6.80(0.8 \mathrm{H}, \mathrm{dq}, J=14.0$, $\left.1.6 \mathrm{~Hz},-\mathrm{CH}_{E}=\mathrm{CHCH}_{3}\right), 6.76\left(0.2 \mathrm{H}, \mathrm{dq}, J=9.2,1.8 \mathrm{~Hz},-\mathrm{CH}_{\mathrm{Z}}=\mathrm{CHCH}_{3}\right), 6.04(0.8 \mathrm{H}, \mathrm{dq}$, $\left.J=13.8,7.0 \mathrm{~Hz},-\mathrm{CH}=\mathrm{CH}_{E} \mathrm{CH}_{3}\right), 5,45\left(0.2 \mathrm{H}\right.$, br quint, $\left.J=7.3 \mathrm{~Hz},-\mathrm{CH}=\mathrm{CH}_{Z} \mathrm{CH}_{3}\right), 1.94(0.4 \mathrm{H}$, $\left.\mathrm{dd}, J=7.4,1.8 \mathrm{~Hz},-\mathrm{CH}=\mathrm{CHCH}_{3}\right), 1.82\left(2.6 \mathrm{H}, \mathrm{dd}, J=6.9,1.8 \mathrm{~Hz},-\mathrm{CH}=\mathrm{CHCH}_{3}\right) ;{ }^{13} \mathrm{C}-\mathrm{NMR}$ $\left(100 \mathrm{MHz}, \mathrm{CDCl}_{3}\right): \delta 145.0,144.8,134.0,133.8,133.6,131.3,128.7,128.5,128.4,127.4,126.7$, 116.7, 114.1, 57.6, 57.4, 14.7, 12.9; HREIMS $m / z$ calcd for $\mathrm{C}_{6} \mathrm{H}_{7} \mathrm{~N}_{2} \mathrm{I}\left(\mathrm{M}^{+}\right)$233.9654, found 233.9652 .

4.3.3. Synthesis of (E/Z)-5-allyl-1-(prop-1-en-1-yl)-1H-pyrazol-4-yl trifluoromethane-sulfonate (12)

A solution of $4 \mathbf{r}(418.9 \mathrm{mg}, 2.6 \mathrm{mmol})$ in 1,2-dimethoxethane (DME, $2 \mathrm{~mL})$ in a sealed vial was heated at $180^{\circ} \mathrm{C}$ for $30 \mathrm{~min}$ under MW irradiation. The reaction mixture was concentrated directly under reduced pressure to give a crude residue that was purified by silica gel column chromatography (eluent:hexane/AcOEt $=2: 1)$ to give $(E / Z)-\mathbf{1 2}(364.3 \mathrm{mg}$, $87 \%)$ as a colorless oil. ${ }^{1} \mathrm{H}-\mathrm{NMR}\left(400 \mathrm{MHz}, \mathrm{CDCl}_{3}\right): \delta 7.30(0.3 \mathrm{H}$, s, pyrazole- $\mathrm{H}), 7.24(0.7 \mathrm{H}$, s, pyrazole- $\mathrm{H}), 6.62\left(0.7 \mathrm{H}, \mathrm{dd}, J=13.7,1.6 \mathrm{~Hz},(E)-\mathrm{NCH}=\mathrm{CHCH}_{3}\right), 6.50(0.3 \mathrm{H}, \mathrm{dd}, J=8.7$, $\left.1.6 \mathrm{~Hz},(\mathrm{Z})-\mathrm{NCH}=\mathrm{CHCH}_{3}\right), 6.15\left(0.7 \mathrm{H}, \mathrm{dq}, J=13.9,6.9 \mathrm{~Hz},(E)-\mathrm{CH}=\mathrm{CHCH}_{3}\right), 5.83-5.94$ $\left(1 \mathrm{H}, \mathrm{m},-\mathrm{CH}_{2} \mathrm{CH}=\mathrm{CH}_{2}\right), 5.54\left(0.3 \mathrm{H}, \mathrm{dq}, J=8.7,7.4 \mathrm{~Hz},(\mathrm{Z})-\mathrm{CH}=\mathrm{CHCH}_{3}\right), 5.12-5.17(1 \mathrm{H}, \mathrm{m}$, $\left.-\mathrm{CH}_{2} \mathrm{CH}=\mathrm{CHH}\right), 5.00(1 \mathrm{H}, \mathrm{br},-\mathrm{OH}), 5.04\left(1 \mathrm{H}, \mathrm{ddd}, J=17.0,7.2,1.1 \mathrm{~Hz},-\mathrm{CH}_{2} \mathrm{CH}=\mathrm{CHH}\right)$, $3.43\left(1.4 \mathrm{H}, \mathrm{d}, J=5.7 \mathrm{~Hz},-\mathrm{CH}_{2} \mathrm{CH}=\mathrm{CH}_{2}\right), 3.38\left(0.6 \mathrm{H}, \mathrm{d}, J=6.1 \mathrm{~Hz},-\mathrm{CH}_{2} \mathrm{CH}=\mathrm{CH}_{2}\right), 1.87$ $\left(0.9 \mathrm{H}, \mathrm{dd}, J=7.2,1.4 \mathrm{~Hz},-\mathrm{CH}=\mathrm{CHCH}_{3}\right), 1.80\left(2.1 \mathrm{H}, \mathrm{dd}, J=6.9,1.2 \mathrm{~Hz},-\mathrm{CH}=\mathrm{CHCH}_{3}\right)$; ${ }^{13} \mathrm{C}-\mathrm{NMR}\left(100 \mathrm{MHz}, \mathrm{CDCl}_{3}\right): \delta 139.2,138.8,133.5,133.3,129.4,129.1,126.9,125.8,124.8$, 124.6, 123.4, 116.7, 116.5, 114.8, 27.0, 26.5, 15.1, 12.9; HREIMS $m / z$ calcd for $\mathrm{C}_{9} \mathrm{H}_{12} \mathrm{~N}_{2} \mathrm{O}\left(\mathrm{M}^{+}\right)$ 164.0950, found 164.0949.

4.3.4. Synthesis of (E/Z)-5-allyl-1-(prop-1-en-1-yl)-1H-pyrazol-4-yl trifluoromethanesulfonate (13)

To a solution of $12(264.7 \mathrm{mg}, 1.6 \mathrm{mmol})$ in $\mathrm{CH}_{2} \mathrm{Cl}_{2}(4 \mathrm{~mL})$ was added triethylamine $\left(0.3 \mathrm{~mL}, 2.4 \mathrm{mmol}, 1.5\right.$ equiv) at $-20^{\circ} \mathrm{C}$ with stirring. After stirring for $10 \mathrm{~min}, \mathrm{Tf}_{2} \mathrm{O}(0.4 \mathrm{~mL}$, $2.4 \mathrm{mmol}, 1.5$ equiv) was added dropwise to the reaction mixture. After stirring at room temperature for another $1 \mathrm{~h}$, the reaction was quenched by the addition of sat. aq. $\mathrm{NH}_{4} \mathrm{Cl}$ $(5 \mathrm{~mL})$ and extracted with $\mathrm{CH}_{2} \mathrm{Cl}_{2}(5 \mathrm{~mL} \times 3)$. The combined organic layers were washed with brine $(5 \mathrm{~mL} \times 2)$, dried over $\mathrm{MgSO}_{4}$, filtered, and evaporated. The obtained residue was purified by silica gel column chromatography (eluent:hexane $/ \mathrm{AcOEt}=2: 1$ ) to give 13 (430.4 mg, 90\%) as an (E/Z) mixture. (Z)-13: Colorless oil; ${ }^{1} \mathrm{H}-\mathrm{NMR}\left(600 \mathrm{MHz}, \mathrm{CDCl}_{3}\right): \delta$ $7.58\left(1 \mathrm{H}\right.$, s, pyrazole-H), $6.54\left(1 \mathrm{H}, \mathrm{dq}, J=8.8,1.8 \mathrm{~Hz},-\mathrm{CH}=\mathrm{CHCH}_{3}\right), 5.79(1 \mathrm{H}, \mathrm{ddt}, J=17.0$, 
10.0, $\left.5.9 \mathrm{~Hz},-\mathrm{CH}_{2} \mathrm{CH}=\mathrm{CH}_{2}\right), 5.71\left(1 \mathrm{H}, \mathrm{dq}, J=8.8,7.3 \mathrm{~Hz},-\mathrm{CH}=\mathrm{CHCH}_{3}\right), 5.17(1 \mathrm{H}, \mathrm{br} \mathrm{dq}$, $\left.J=10.3,1.8 \mathrm{~Hz},-\mathrm{CH}_{2} \mathrm{CH}=\mathrm{CHH}\right), 5.06\left(1 \mathrm{H}, \mathrm{br} \mathrm{dq}, J=17.0,1.8 \mathrm{~Hz},-\mathrm{CH}_{2} \mathrm{CH}=\mathrm{CHH}\right), 3.42(2 \mathrm{H}$, $\left.\mathrm{dt}, J=6.1,1.6 \mathrm{~Hz},-\mathrm{CH}_{2} \mathrm{CH}=\mathrm{CH}_{2}\right), 1.87\left(3 \mathrm{H}, \mathrm{dd}, J=7.4,1.8 \mathrm{~Hz},-\mathrm{CH}=\mathrm{CHCH}_{3}\right) ;{ }^{13} \mathrm{C}-\mathrm{NMR}$ $\left(100 \mathrm{MHz}, \mathrm{CDCl}_{3}\right): \delta 132.4,131.4,131.2,130.9,124.4,123.9,118.7\left(\mathrm{q}, J=321.43 \mathrm{~Hz},-\mathrm{CF}_{3}\right)$, 118.2, 27.2, 12.9; HREIMS $m / z$ calcd for $\mathrm{C}_{10} \mathrm{H}_{11} \mathrm{~F}_{3} \mathrm{~N}_{2} \mathrm{O}_{3} \mathrm{~S}\left(\mathrm{M}^{+}\right)$296.0442, found 296.0443 . (E)-13: colorless oil; ${ }^{1} \mathrm{H}-\mathrm{NMR}\left(600 \mathrm{MHz}, \mathrm{CDCl}_{3}\right): \delta 7.58(1 \mathrm{H}, \mathrm{s}$, pyrazole-H), $6.54(1 \mathrm{H}, \mathrm{dq}$, $\left.J=8.8,1.8 \mathrm{~Hz},-\mathrm{CH}=\mathrm{CHCH}_{3}\right), 5.79\left(1 \mathrm{H}, \mathrm{ddt}, J=17.0,10.0,5.9 \mathrm{~Hz},-\mathrm{CH}_{2} \mathrm{CH}=\mathrm{CH}_{2}\right), 5.83(1 \mathrm{H}$, $\left.\mathrm{dq}, J=8.8,7.3 \mathrm{~Hz},-\mathrm{CH}=\mathrm{CHCH}_{3}\right), 5.21\left(1 \mathrm{H}, \mathrm{br} \mathrm{dq}, J=10.3,1.7 \mathrm{~Hz},-\mathrm{CH}_{2} \mathrm{CH}=\mathrm{CHH}\right) 5.06$ $\left(1 \mathrm{H}, \mathrm{br} \mathrm{dq}, J=17.0,1.8 \mathrm{~Hz},-\mathrm{CH}_{2} \mathrm{CH}=\mathrm{CHH}\right), 3.47\left(2 \mathrm{H}, \mathrm{dt}, J=5.9,1.8 \mathrm{~Hz},-\mathrm{CH}_{2} \mathrm{CH}=\mathrm{CH}_{2}\right)$, $1.84\left(3 \mathrm{H}, \mathrm{dd}, J=7.0,1.9 \mathrm{~Hz},-\mathrm{CH}=\mathrm{CHCH}_{3}\right) ;{ }^{13} \mathrm{C}-\mathrm{NMR}\left(100 \mathrm{MHz}, \mathrm{CDCl}_{3}\right): \delta 131.6131 .4$, 131.0, 130.9, 124.3, $118.7\left(\mathrm{q}, J=321.3 \mathrm{~Hz},-\mathrm{CF}_{3}\right), 118.3,117.9,26.7,15.0 ;$ HREIMS $m / z$ calcd for $\mathrm{C}_{10} \mathrm{H}_{11} \mathrm{~F}_{3} \mathrm{~N}_{2} \mathrm{O}_{3} \mathrm{~S}\left(\mathrm{M}^{+}\right)$296.0442, found 296.0443 .

\subsubsection{Synthesis of $4 H$-pyrrolo [1,2-b]pyrazol-3-yl trifluoromethanesulfonate (14)}

To a solution of $\mathbf{1 3}(50.0 \mathrm{mg}, 0.17 \mathrm{mmol})$ in $\mathrm{CH}_{2} \mathrm{Cl}_{2}(2 \mathrm{~mL})$ were added Grubbs ${ }^{2 \text { nd }}$ catalyst ( $7.1 \mathrm{mg}, 0.0085 \mathrm{mmol}, 5 \mathrm{~mol} \%)$ and $\mathrm{CuI}(0.8 \mathrm{mg}, 0.0042 \mathrm{mmol}, 2.5 \mathrm{~mol} \%)$. The sealed reaction mixture was heated at $80^{\circ} \mathrm{C}$ for $1 \mathrm{~h}$ under MW irradiation. The solvent was removed from the mixture under reduced pressure to give a crude material that was purified by silica gel column chromatography (eluent:hexane/AcOEt $=4: 1$ ) to afford $\mathbf{1 4}$ (27.0 mg, 63\%). 14: Colorless oil; ${ }^{1} \mathrm{H}-\mathrm{NMR}\left(400 \mathrm{MHz}, \mathrm{CDCl}_{3}\right): \delta 7.55(1 \mathrm{H}, \mathrm{s}$, pyrazole-H), $7.21\left(1 \mathrm{H}, \mathrm{dt}, 4.1,2.2 \mathrm{~Hz},-\mathrm{CH}_{2} \mathrm{CH}=\mathrm{CH}-\right), 6.08\left(1 \mathrm{H}, \mathrm{m},-\mathrm{CH}_{2} \mathrm{CH}=\mathrm{CH}-\right), 3.55(2 \mathrm{H}, \mathrm{br} \mathrm{t}, J=$ $\left.2.3 \mathrm{~Hz}, \mathrm{ArCH}_{2} \mathrm{CH}=\mathrm{CH}-\right) ;{ }^{13} \mathrm{C}-\mathrm{NMR}\left(100 \mathrm{MHz}, \mathrm{CDCl}_{3}\right): \delta 135.0,134.0,130.0,129.0,118.6$ $\left(\mathrm{q}, J=322.0 \mathrm{~Hz},-\mathrm{CF}_{3}\right), 118.4,30.3$; HREIMS $\mathrm{m} / \mathrm{z}$ calcd for $\mathrm{C}_{7} \mathrm{H}_{5} \mathrm{~F}_{3} \mathrm{~N}_{2} \mathrm{O}_{3} \mathrm{~S}\left(\mathrm{M}^{+}\right)$253.9973, found 253.9977 .

4.3.6. Synthesis of 5,6-dihydro-4H-pyrrolo[1,2-b]pyrazol-3-yl trifluoromethanesulfonate (6)

To a solution $14(50.0 \mathrm{mg}, 0.20 \mathrm{mmol})$ in $\mathrm{MeOH}(5 \mathrm{~mL})$ was added $\mathrm{Pd} / \mathrm{C}(5.00 \mathrm{mg}$, $10 \mathrm{~mol} \%$ ). The mixture was stirred for $24 \mathrm{~h}$ at room temperature under hydrogen gas at $1 \mathrm{~atm}$. After removal of $\mathrm{Pd} / \mathrm{C}$ by filtration, the solvent was evaporated to give a crude mixture that was purified by silica gel column chromatography (eluent:hexane $/ \mathrm{AcOEt}=8: 1$ ) to afford 6 (45.6 mg, 90\%) [22,23].

\subsection{Synthesis of Withasomnine Homolog 15 (Scheme 3) \\ 4.4.1. Synthesis of 1-allyl-4-iodo- $1 H$-pyrazole (2d)}

To a solution of 1 (300.0 mg, $1.5 \mathrm{mmol})$ in acetone $(9 \mathrm{~mL})$ was added $20 \% \mathrm{NaOH}$ aq. ( $6 \mathrm{~mL}$, excess) with stirring, followed by allyl bromide $(0.3 \mathrm{~mL}, 3.0 \mathrm{mmol})$, and the reaction mixture was stirred at $\mathrm{rt}$ for $1 \mathrm{~h}$. After quenching with sat. aq. $\mathrm{NH}_{4} \mathrm{Cl}(10 \mathrm{~mL})$, the mixture was extracted with $\mathrm{CH}_{2} \mathrm{Cl}_{2}(20 \mathrm{~mL} \times 3)$, and the combined organic layers were washed with brine $(5 \mathrm{~mL} \times 2)$, dried over $\mathrm{MgSO}_{4}$, and filtered. The solvent was removed under reduced pressure to give a crude residue that was purified by silica gel column chromatography (eluent:hexane/ $\mathrm{AcOEt}=3: 1)$ to afford $\mathbf{2 d}(373.9 \mathrm{mg}, 88 \%)$. 2d: Colorless oil; ${ }^{1} \mathrm{H}-\mathrm{NMR}\left(400 \mathrm{MHz}, \mathrm{CDCl}_{3}\right): \delta 7.50(1 \mathrm{H}, \mathrm{s}$, pyrazole-H), $7.42(1 \mathrm{H}, \mathrm{s}$, pyrazole-H), 5.72 $\left(1 \mathrm{H}, \mathrm{ddt}, J=17.4,9.9,7.0 \mathrm{~Hz},-\mathrm{CH}_{2} \mathrm{CH}=\mathrm{CH}_{2}\right), 5.08(1 \mathrm{H}, \mathrm{br} \mathrm{d}, J=17.4 \mathrm{~Hz},-\mathrm{CH}=\mathrm{CHH})$, $5.08(1 \mathrm{H}$, br d, $J=9.9 \mathrm{~Hz},-\mathrm{CH}=\mathrm{CHH}), 4.18\left(2 \mathrm{H}, \mathrm{t}, J=7.0 \mathrm{~Hz}, \mathrm{NHCH}_{2} \mathrm{CH}_{2}-\right), 2.60(2 \mathrm{H}, \mathrm{br}$, $\left.J=7.0 \mathrm{~Hz},-\mathrm{CH}_{2} \mathrm{CH}_{2} \mathrm{CH}=\right) ;{ }^{13} \mathrm{C}-\mathrm{NMR}\left(100 \mathrm{MHz}, \mathrm{CDCl}_{3}\right) \delta 144.2,133.7,133.5,117.9$, 55.6, $52.0,34.5$ (one aromatic or olefinic carbon signal is overlapped); HREIMS $\mathrm{m} / \mathrm{z}$ calcd for $\mathrm{C}_{7} \mathrm{H}_{9} \mathrm{~N}_{2} \mathrm{I}\left(\mathrm{M}^{+}\right)$247.9810, found 247.9810 .

\subsubsection{Synthesis of 5-allyl-1-(but-3-en-1-yl)-4-hydroxy-1H-pyrazole (16)}

A solution of $4 \mathbf{t}(81.8 \mathrm{mg}, 0.46 \mathrm{mmol})$ in DME $(2 \mathrm{~mL})$ in a sealed vial was heated at $200{ }^{\circ} \mathrm{C}$ for $1 \mathrm{~h}$ under MW irradiation. The reaction mixture was concentrated directly under reduced pressure to give a crude residue that was purified by silica gel column chromatography (eluent:hexane / AcOEt $=2: 1)$ to give $\mathbf{1 6}(70.0 \mathrm{mg}, 81 \%) . \mathbf{1 6}$ : Colorless oil; ${ }^{1} \mathrm{H}-\mathrm{NMR}\left(400 \mathrm{MHz}, \mathrm{CDCl}_{3}\right): \delta 7.12(1 \mathrm{H}, \mathrm{s}$, pyrazole-H), $6.91(1 \mathrm{H}, \mathrm{br} \mathrm{s},-\mathrm{OH}), 5.87(1 \mathrm{H}, \mathrm{ddt}$, 
$\left.J=17.0,10.0,5.8 \mathrm{~Hz},-\mathrm{OCH}_{2} \mathrm{CH}=\mathrm{CH}_{2}\right), 5.71\left(1 \mathrm{H}, \mathrm{ddt}, J=17.0,10.4,7.0,-\mathrm{CH}_{2} \mathrm{CH}=\mathrm{CH}_{2}\right)$, $5.11(1 \mathrm{H}, \mathrm{dq}, J=10.2,1.4 \mathrm{~Hz},-\mathrm{CH}=\mathrm{CHH}), 5.01-5.07(3 \mathrm{H}$, overlapped. $3 \times-\mathrm{CH}=\mathrm{CHH})$, $3.98\left(2 \mathrm{H}, \mathrm{t}, J=7.4 \mathrm{~Hz}, \mathrm{NCH}_{2} \mathrm{CH}_{2}-\right), 3.39\left(2 \mathrm{H}, \mathrm{dt}, J=5.8,1.5 \mathrm{~Hz}, \mathrm{ArCH}_{2} \mathrm{CH}=\right), 2.49(2 \mathrm{H}$, br q, $\left.J=7.2 \mathrm{~Hz},-\mathrm{CH}_{2} \mathrm{CH}_{2} \mathrm{CH}=\right) ;{ }^{13} \mathrm{C}-\mathrm{NMR}\left(100 \mathrm{MHz}, \mathrm{CDCl}_{3}\right): \delta 138.3,134.1,133.7,127.6$, 126.0, 116.6, 116.4, 48.9, 34.5, 27.0; HREIMS $m / z$ calcd for $\mathrm{C}_{10} \mathrm{H}_{14} \mathrm{~N}_{2} \mathrm{O}\left(\mathrm{M}^{+}\right)$178.1106, Found 178.1104.

4.4.3. Synthesis of 5-allyl-1-(but-3-en-1-yl)-1H-pyrazol-4-yl trifluoromethanesulfonate (17)

To a solution of $16(70.0 \mathrm{mg}, 0.39 \mathrm{mmol})$ in $\mathrm{CH}_{2} \mathrm{Cl}_{2}(10 \mathrm{~mL})$ was added triethylamine (0.06 mL, $0.43 \mathrm{mmol}, 1.1$ equiv) at $-20^{\circ} \mathrm{C}$ with stirring. After stirring for $10 \mathrm{~min}, \mathrm{Tf}_{2} \mathrm{O}$ $(0.1 \mathrm{~mL}, 0.6 \mathrm{mmol}, 1.5$ equiv) was added dropwise to the reaction mixture. After stirring at room temperature for another $1 \mathrm{~h}$, the reaction was quenched by the addition of sat. aq. $\mathrm{NH}_{4} \mathrm{Cl}(5 \mathrm{~mL})$ and extracted with $\mathrm{CH}_{2} \mathrm{Cl}_{2}(10 \mathrm{~mL} \times 3)$. The combined organic layers were washed with brine $(5 \mathrm{~mL} \times 2)$, dried over $\mathrm{MgSO}_{4}$, filtered, and concentrated. The obtained residue was purified by silica gel column chromatography (eluent:hexane $/ \mathrm{AcOEt}=2: 1$ ) to give 17 (100.6 mg, 83\%). 17: Colorless oil; ${ }^{1} \mathrm{H}-\mathrm{NMR}\left(400 \mathrm{MHz}, \mathrm{CDCl}_{3}\right): \delta 7.48(1 \mathrm{H}, \mathrm{s}$, pyrazole- $\mathrm{H}), 5.88-5.67\left(2 \mathrm{H}\right.$, overlapped, $\left.2 \times-\mathrm{CH}_{2} \mathrm{CH}=\mathrm{CH}_{2}\right), 5.20(1 \mathrm{H}, \mathrm{dq}, J=10.2,1.2 \mathrm{~Hz}$, $-\mathrm{CH}=\mathrm{CHH}), 5.12-5.03(3 \mathrm{H}$, overlapped. $3 \times-\mathrm{CH}=\mathrm{CHH}), 4.06\left(2 \mathrm{H}, \mathrm{t}, J=7.3 \mathrm{~Hz}, \mathrm{NCH}_{2} \mathrm{CH}_{2}-\right)$, $3.44\left(2 \mathrm{H}, \mathrm{dt}, J=5.8,1.6 \mathrm{~Hz}, \mathrm{ArCH}_{2} \mathrm{CH}=\right), 2.57\left(2 \mathrm{H}\right.$, br q, $\left.J=7.4 \mathrm{~Hz},-\mathrm{CH}_{2} \mathrm{CH}_{2} \mathrm{CH}=\right)$; ${ }^{13} \mathrm{C}-\mathrm{NMR}\left(100 \mathrm{MHz}, \mathrm{CDCl}_{3}\right): \delta 133.2,131.5,130.6,129.7,118.4\left(\mathrm{q}, J_{\mathrm{C}-\mathrm{F}}=321.2 \mathrm{~Hz}\right), 117.8$, 117.7, 100.5, 49.4, 33.8, 26.9; HREIMS $m / z$ calcd for $\mathrm{C}_{11} \mathrm{H}_{13} \mathrm{~F}_{3} \mathrm{~N}_{2} \mathrm{O}_{3} \mathrm{~S}\left(\mathrm{M}^{+}\right)$310.0599, Found 310.0598 .

4.4.4. Synthesis of 7,8-dihydro-4H-pyrazolo[1,5-a]azepin-3-yl trifluoromethanesulfonate (18)

To a solution of $\mathbf{1 7}(99.1 \mathrm{mg}, 0.32 \mathrm{mmol})$ in $\mathrm{CH}_{2} \mathrm{Cl}_{2}(2 \mathrm{~mL})$ were added Grubbs ${ }^{2 \text { nd }}$ catalyst (13 mg, $0.016 \mathrm{mmol}, 5 \mathrm{~mol} \%)$ and CuI (1.0 mg, $0.005 \mathrm{mmol}, 2.5 \mathrm{~mol} \%)$. The sealed reaction mixture was heated at $80^{\circ} \mathrm{C}$ for $1 \mathrm{~h}$ under MW irradiation. Solvent was removed under reduced pressure to give a crude mixture that was purified by silica gel column chromatography (eluent:hexane/ $\mathrm{AcOEt}=4: 1)$ to afford $18(65.1 \mathrm{mg}, 72 \%)$. 18: Amorphous solid; mp 72-75 ${ }^{\circ} \mathrm{C} ;{ }^{1} \mathrm{H}-\mathrm{NMR}\left(400 \mathrm{MHz}, \mathrm{CDCl}_{3}\right): \delta 7.38(1 \mathrm{H}, \mathrm{s}$, pyrazole-H), 5.77-5.70 $\left(2 \mathrm{H}, \mathrm{m}, 2 \times-\mathrm{CH}=\mathrm{CHCH}_{2}-\right), 4.46\left(2 \mathrm{H}, \mathrm{dd}, J=5.6,4.5 \mathrm{~Hz}, \mathrm{NCH}_{2} \mathrm{CH}_{2}-\right), 3.47-3.45(2 \mathrm{H}, \mathrm{m}$, $\left.\mathrm{ArCH}_{2} \mathrm{CH}=\right), 2.49-2.45\left(2 \mathrm{H}, \mathrm{m},-\mathrm{CH}_{2} \mathrm{CH}_{2} \mathrm{CH}=\right) ;{ }^{13} \mathrm{C}-\mathrm{NMR}\left(100 \mathrm{MHz}, \mathrm{CDCl}_{3}\right): \delta 134.0,129.8$, 129.16, 129.12, 122.4, $118.6\left(\mathrm{q}, J_{\mathrm{C}-\mathrm{F}}=321.2 \mathrm{~Hz}\right), 50.9,27.8,21.7$; HREIMS $m / z$ calcd for $\mathrm{C}_{9} \mathrm{H}_{9} \mathrm{~F}_{3} \mathrm{~N}_{2} \mathrm{O}_{3} \mathrm{~S}\left(\mathrm{M}^{+}\right)$282.0286, found 282.0282.

\subsubsection{Synthesis of 3-phenyl-7,8-dihydro-4H-pyrazolo[1,5-a]azepine (19)}

To a solution of $18(54.7 \mathrm{mg}, 0.19 \mathrm{mmol})$ in DME $/ \mathrm{H}_{2} \mathrm{O}=9: 1(5 \mathrm{~mL})$ in a MW vial were added XPhos (9.2 mg, $0.019 \mathrm{mmol}, 10 \mathrm{~mol} \%)$, Pd(dba) 2 (11.0 mg, $0.019 \mathrm{mmol}, 10 \mathrm{~mol} \%)$, cesium carbonate ( $126.4 \mathrm{mg}, 0.38 \mathrm{mmol}, 2.0$ eq.), and phenylboronic acid $(50.0 \mathrm{mg}, 0.41 \mathrm{mmol}$, 2.0 eq.). The sealed vial was heated under MW irradiation at $130^{\circ} \mathrm{C}$ for $1 \mathrm{~h}$. The cooled reaction mixture was quenched with sat. aq. $\mathrm{NH}_{4} \mathrm{Cl}$ solution $(40 \mathrm{~mL})$ and extracted with $\mathrm{CH}_{2} \mathrm{Cl}_{2}(10 \mathrm{~mL} \times 3)$. The combined organic layers were dried over $\mathrm{MgSO}_{4}$, filtered, and the solvent was removed under reduced pressure to give a crude residue that was purified by silica gel column chromatography (eluent:hexane/AcOEt $=2: 1)$ to afford $19(35.4 \mathrm{mg}, 87 \%)$. 19: Oil; ${ }^{1} \mathrm{H}-\mathrm{NMR}\left(400 \mathrm{MHz}, \mathrm{CDCl}_{3}\right): \delta 7.49(1 \mathrm{H}, \mathrm{s}$, pyrazole-H), $7.42-7.34(2 \mathrm{H}, \mathrm{m}, \mathrm{Ph}-\mathrm{H})$, 7.34-7.26 (3H, m, Ph-H), 5.73-5.56 (2H, m, $\left.-\mathrm{NCH}_{2} \mathrm{CH}=\mathrm{CH}-,-\mathrm{CH}=\mathrm{CHCH}_{2}-\right), 4.54-4.51(\mathrm{~m}$, 2H), 3.61-3.59 (2H, m), 2.50-2.46 (2H, m); ${ }^{13} \mathrm{C}-\mathrm{NMR}\left(100 \mathrm{MHz}, \mathrm{CDCl}_{3}\right): \delta 138.9,136.8$, $133.6,129.1,128.9,128.6,128.2,126.3,123.8,120.7,49.5,28.4,23.1$; HREIMS $m / z$ calcd for $\mathrm{C}_{14} \mathrm{H}_{14} \mathrm{~N}_{2}\left(\mathrm{M}^{+}\right)$210.1157, found 210.1156.

\subsubsection{Synthesis of 3-phenyl-5,6,7,8-tetrahydro-4H-pyrazolo[1,5-a]azepine (15)}

To a solution of $19(3.0 \mathrm{mg}, 0.014 \mathrm{mmol})$ in $\mathrm{MeOH}(10 \mathrm{~mL})$ was added $\mathrm{Pd} / \mathrm{C}(0.2 \mathrm{mg}$, $10 \mathrm{~mol} \%$ ). The mixture was stirred overnight at room temperature under hydrogen gas at $1 \mathrm{~atm}$. After removal of $\mathrm{Pd} / \mathrm{C}$ by filtration, the solvent was evaporated to give a crude 
residue that was purified by column chromatography (eluent:hexane $/ \mathrm{AcOEt}=4: 1$ ) to afford $15(2.8 \mathrm{mg}, 92 \%)$. 15: ${ }^{1} \mathrm{H}-\mathrm{NMR}\left(400 \mathrm{MHz}, \mathrm{CDCl}_{3}\right): \delta 7.44(1 \mathrm{H}, \mathrm{s}$, pyrazole-H), 7.37-7.42 (2H, m, Ph-H), 7.33-7.25 (3H, m, Ph-H), 4.35-4.30 (2H, m, - $\left.\mathrm{NCH}_{2} \mathrm{CH}_{2}-\right), 2.91-2.86$ $\left(2 \mathrm{H}, \mathrm{m}, \mathrm{ArCH}_{2} \mathrm{CH}_{2}-\right), 1.93-1.86(2 \mathrm{H}, \mathrm{m}), 1.86-1.80(2 \mathrm{H}, \mathrm{m}), 1.74-1.67(2 \mathrm{H}, \mathrm{m})$; lit. ${ }^{1} \mathrm{H}-\mathrm{NMR}$ $\left(400 \mathrm{MHz}, \mathrm{CDCl}_{3}\right): \delta 7.43(1 \mathrm{H}, \mathrm{s}$, pyrazole- $\mathrm{H}), 7.40-7.26(5 \mathrm{H}, \mathrm{m}, \mathrm{Ph}-\mathrm{H}), 4.33-4.31(2 \mathrm{H}, \mathrm{m}$, - $\left.\mathrm{NCH}_{2} \mathrm{CH}_{2}-\right), 2.89-2.86\left(2 \mathrm{H}, \mathrm{m}, \mathrm{ArCH}_{2} \mathrm{CH}_{2}-\right)$ 1.89-1.79 (4H, m), 1.75-1.67 (2H, m) [26]; ${ }^{13} \mathrm{C}-\mathrm{NMR}\left(100 \mathrm{MHz}, \mathrm{CDCl}_{3}\right): \delta 140.7,136.6,134.1,128.6,128.3,126.1,121.0,53.4,31.0$, 28.0, 26.8, 24.4; lit. ${ }^{13} \mathrm{C}-\mathrm{NMR}\left(100 \mathrm{MHz}, \mathrm{CDCl}_{3}\right): \delta 140.7,136.5,134.1,128.5,128.3,126.1$, 121.0, 53.3, 30.9, 28.0, 26.8, 24.4; HREIMS $m / z$ calcd for $\mathrm{C}_{14} \mathrm{H}_{16} \mathrm{~N}_{2}\left(\mathrm{M}^{+}\right)$212.1313, found $212.1312[26]$.

\subsubsection{Synthesis of $\mathbf{4 t}$ from $\mathbf{4 b}$}

To a solution of $4 \mathbf{b}(48.6 \mathrm{mg}, 0.39 \mathrm{mmol})$ in acetone $(1 \mathrm{~mL})$ was added $20 \% \mathrm{NaOH}$ aq. ( $0.12 \mathrm{mg}, 1.5 \mathrm{eq}$.) with stirring, followed by 1-bromo-3-butene $(0.08 \mathrm{~mL}, 0.08 \mathrm{mmol}, 2 \mathrm{eq}$.$) .$ The reaction mixture was heated at $80^{\circ} \mathrm{C}$ for $10 \mathrm{~min}$ under $\mathrm{MW}$ irradiation. After addition of sat. aq. $\mathrm{NH}_{4} \mathrm{Cl}(1 \mathrm{~mL})$ to the reaction mixture, it was extracted with $\mathrm{CH}_{2} \mathrm{Cl}_{2}(10 \mathrm{~mL} \times 3)$ and the combined organic layers were washed with brine $(5 \mathrm{~mL} \times 2)$, dried over $\mathrm{MgSO}_{4}$, and filtered. The solvent was removed under reduced pressure to give a crude residue that was purified by silica gel column chromatography (eluent:hexane $/ \mathrm{AcOEt}=3: 1$ ) to afford $4 \mathbf{t}(48.1 \mathrm{mg}, 69 \%)$.

Supplementary Materials: Figures S1-S48: ${ }^{1} \mathrm{H}$ - and ${ }^{13} \mathrm{C}-\mathrm{NMR}$ spectra of compounds $2 \mathrm{c}, \mathbf{2 d}, \mathbf{4 c}, 4 \mathrm{~d}$, $4 \mathrm{e}, 4 \mathrm{f}, 4 \mathrm{~g}, 4 \mathrm{i}, 4 \mathrm{k}, 4 \mathrm{l}, 4 \mathrm{~m}, 4 \mathrm{n}, 4 \mathrm{o}, 4 \mathrm{r}, 4 \mathrm{~s}, 4 \mathrm{t}$, and 12-19.

Author Contributions: Y.U. conceived and designed experiments. Y.K., N.K., T.T., J.O., K.N., A.N. and Y.T. performed experimental work. N.H., S.H. and H.Y. made discussions and suggestions on this work and wrote the manuscript with Y.U. All authors have read and agreed to the published version of the manuscript.

Funding: This research received no external funding.

Acknowledgments: We thank K. Minoura and Fujitake for the NMR and MS measurements, respectively. K. Sumimoto of our laboratory is also appreciated for providing inspiration and suggestions on the improved synthesis of withasomnine at an early stage.

Conflicts of Interest: The authors declare no conflict of interest.

Sample Availability: Samples of the compounds are not available from the authors.

\section{References}

1. Fustero, S.; Sánchez-Roselló, M.; Barrio, P.; Simón-Fuentes, A. From 2000 to Mid-2010: A fruitful decade for the synthesis of pyrazoles. Chem. Rev. 2011, 111, 6984-7034. [CrossRef]

2. Ansari, A.; Ali, A.; Asif, M.; Shamsuzzaman, S. Review: Biologically active pyrazole derivatives. New J. Chem. 2017, 41, 16-41. [CrossRef]

3. Karrouchi, K.; Radi, S.; Ramli, Y.; Taoufik, J.; Mabkhot, Y.N.; Al-Aizari, F.A.; Ansar, M. Synthesis and pharmacological activities of pyrazole derivatives: A review. Molecules 2018, 23, 134. [CrossRef]

4. Brown, A.W. Recent developments in the chemistry of pyrazoles. Adv. Heterocycl. Chem. 2018, 126, 55-107.

5. Usami, Y.; Tatsui, Y.; Yoneyama, H.; Harusawa, S. C4-Alkylamination of C4-halo-1H-1-tritylpyrazoles using Pd(dba) 2 or CuI. Molecules 2020, 25, 4634. [CrossRef]

6. Ray, R.; Hartwig, J.F. Oxalohydrazide ligands for copper-catalyzed C-O coupling reactions with high turnover numbers. Angew. Chem. Int. Ed. 2020, 133, 8284-8292. [CrossRef]

7. Vijeta, A.; Casadevall, C.; Roy, S.; Reisner, E. Visible-light promoted C-O bond formation with an integrated carbon nitride-nickel heterogeneous photocatalyst. Angew. Chem. Int. Ed. 2021, 60, 8494-8499. [CrossRef] [PubMed]

8. Vorogushin, A.V.; Huang, X.; Buchwald, S.L. Use of tunable ligands allows for intermolecular Pd-catalyzed C-O Bond formation. J. Am. Chem. Soc. 2005, 127, 8146-8149. [CrossRef] [PubMed]

9. Maiti, D.; Buchwald, S.L. Orthogonal Cu- and Pd-based catalyst system for the O- and N-arylation of aminophenols. J. Am. Chem. Soc. 2009, 131, 17423-17429. [CrossRef] [PubMed]

10. Zhang, H.; Ruiz-Castillo, P.; Buchwald, S.L. Palladium-catalyzed C-O cross-coupling of primary alcohols. Org. Lett. 2018, 20, 1580-1583. [CrossRef] [PubMed] 
11. Gowrisankar, S.; Sergeev, A.G.; Anbarasan, P.; Spannenberg, A.; Neumann, H.; Beller, M. A general and efficient catalyst for palladium-catalyzed C-O coupling reactions of aryl halides with primary alcohols. J. Am. Chem. Soc. 2010, 132, 11592-11598. [CrossRef]

12. Maiti, D.; Buchwald, S.L. Cu-catalyzed arylation of phenols: Synthesis of sterically hindered and heteroaryl diaryl ethers. J. Org. Chem. 2010, 75, 1791-1794. [CrossRef] [PubMed]

13. Nayak, M.; Batra, S. Copper-Catalyzed Cascade Reactions of Substituted 4-Iodopyrazolecarbaldehydes with 1,2-Phenylenediamines and 2-Aminophenols. Adv. Synth. Catal. 2020, 352, 3431-3437. [CrossRef]

14. Wong, D.M.; Li, J.; Chen, Q.H.; Han, Q.; Mutunga, J.M.; Wysinski, A.; Anderson, T.D. Select small core structure carbamates exhibit high contact toxicity to "carbamate-resistant" strain malaria mosquitoes, Anopheles gambiae (Akron). PLoS ONE 2012, 7, e46712. [CrossRef] [PubMed]

15. MacDonald, E.; Ihalainen, E.; Pispa, J.P. Pharmacological and toxicological properties of by 4-hydroxypyrazole, a metabolite of pyrazole. Acta Pharmacol. Toxicol. 1981, 48, 418-423. [CrossRef] [PubMed]

16. Cornell, N.W.; Hansch, C.; Kim, K.H.; Henegar, K. The inhibition of alcohol dehydrogenase in vitro and in isolated hepatocytes by 4-substituted pyrazoles. Arch. Biochem. Biophys. 1983, 227, 81-90. [CrossRef]

17. Sinclair, J.; Cornell, N.W.; Zaitlin, L.; Hansch, C. Induction of cytochrome P-450 by alcohols and 4-substituted pyrazoles: Comparison of structure-activity relationships. Biochem. Pharmacol. 1986, 35, 707-710. [CrossRef]

18. Jones, J.P.; Joswig-Jones, C.A.; Hebner, M.; Chu, Y.; Koop, D.R. The effects of nitrogen-heme-iron coordination on substrate affinities for cytochrome P450 2E1. Chem. Biol. Interact. 2011, 193, 50-56. [CrossRef] [PubMed]

19. Walworth, B.L. Fungicidal Use of 4-alkoxypyrazoles. U.S. (1976) Patent No. US 4000301 A 19761228, 14 November 1975.

20. Matsuo, J.; Takaya, M.; Maki, Y. 1-Phenyl-4-alkoxypyrazole-5-carboxylic Acid Esters. Jpn. Kokai Tokkyo Koho (1972) Patent No. JP 47042665 A, 16 December 1972.

21. Munier-Lehmann, H.; Lucas-Hourani, M.; Guillou, S.; Helynck, O.; Zanghi, G.; Noel, A.; Tangy, F.; Vidalain, P.O.; Janin, Y.L. Original 2-(3-Alkoxy-1H-pyrazol-1-yl)pyrimidine derivatives as inhibitors of human dihydroorotate dehydrogenase (DHODH). J. Med. Chem. 2015, 58, 860-877. [CrossRef]

22. Ichikawa, H.; Watanabe, R.; Fujino, Y.; Usami, Y. Divergent synthesis of withasomnines via synthesis of 4-hydroxy-1H-pyrazoles and Claisen rearrangement of their 4-O-allylethers. Tetrahedron Lett. 2011, 52, 4448-4451. [CrossRef]

23. Usami, Y.; Watanabe, R.; Fujino, Y.; Shibano, M.; Ishida, C.; Yoneyama, H.; Harusawa, S.; Ichikawa, H. Divergent synthesis and evaluation of inhibitory activities against Cyclooxygenases- 1 and -2 of natural withasomnines and analogues. Chem. Pharm. Bull. 2012, 60, 1550-1560. [CrossRef]

24. Usami, Y.; Tatsui, Y.; Sumimoto, K.; Miyamoto, A.; Koito, N.; Yoneyama, H.; Harusawa, S. 3-Trifluoromethansulfonyloxy-4,7dihidropyrazolopyridine via ring-closing metathesis: Synthesis and transformation to withasomnine homologs. Heterocycles 2021, 103, 284-299. [CrossRef]

25. Allin, S.M.; Barton, W.R.S.; Bowman, W.R.; McInally, T. Radical cyclisation onto pyrazoles: Synthesis of withasomnine. Tetrahedron Lett. 2002, 43, 4191-4193. [CrossRef]

26. Allin, S.M.; Barton, W.R.S.; Russell Bowman, W.; Bridge (née Mann), E.; Elsegood, M.R.J.; McInally, T.; McKee, V. Bus 3 SnHmediated radical cyclisation onto azoles. Tetrahedron 2008, 64, 7745-7758. [CrossRef]

27. Xia, T.; Hu, Z.; Ji, W.; Zhang, S.; Shi, H.; Liu, C.; Pang, B.; Liu, G.; Liao, X. Synthesis of withasomnine and pyrazole derivatives via intramolecular dehydrogenative cyclization, as well as biological evaluation of withasomnine-based scaffolds. Org. Chem. Front. 2018, 5, 850-854. [CrossRef]

28. Wube, A.A.; Wenzig, E.M.; Gibbons, S.; Asres, K.; Bauer, R.; Bucar, F. Constituents of the stem bark of Discopodium penninervium and their $\mathrm{LTB}_{4}$ and COX-1 and -2 inhibitory activities. PhytoChemistry 2008, 69, 982-987. [CrossRef]

29. Usami, Y.; Kohno, A.; Yoneyama, H.; Harusawa, S. Synthesis of dihydrooxepino[3,2-c]pyrazoles via Claisen rearrangement and ring-closing metathesis from 4-allyloxy-1H-pyrazoles. Molecules 2018, 23, 592. [CrossRef]

30. Usami, Y.; Sumimoto, K.; Kishima, A.; Tatsui, Y.; Yoneyama, H.; Harusawa, S. Synthesis of dihydropyrano[3,2-c]pyrazoles via double bond migration and ring-closing metathesis. Molecules 2019, 24, 296. [CrossRef]

31. Usami, Y.; Tsujiuchi, Y.; Machiya, Y.; Chiba, A.; Ikawa, T.; Yoneyama, H.; Harusawa, S. Synthetic challenges in the construction of 8- to 10-Membered pyrazole-fused rings via ring-closing metathesis. Heterocycles 2020, 101, 496-511. [CrossRef]

32. Kadoma, Y.; Murakami, Y.; Ogiwara, T.; Machino, M.; Yokoe, I.; Fujisawa, S. Radical-scavenging activity and cytotoxicity of p-methoxyphenol and p-cresol dimers. Molecules 2010, 15, 1103-1112. [CrossRef]

33. Li, X.B.; Chen, G.Y.; Liu, R.J.; Zheng, C.J.; Song, X.M.; Han, C.R. A new biphenyl derivative from the mangrove endophytic fungus Phomopsis longicolla HL-2232. Nat. Prod. Res. 2017, 31, 2264-2267. [CrossRef] [PubMed]

34. Allen, S.E.; Walvoord, R.R.; Padilla-Salinas, R.; Kozlowski, M.C. Aerobic copper-catalyzed organic reactions. Chem. Rev. 2013, 113, 6234-6458. [CrossRef] [PubMed]

35. Kamal, F.; Colombel-Rouen, S.; Dumas, A.; Guégan, J.P.; Roisnel, T.; Dorcet, V.; Baslé, O.; Rouen, M.; Mauduit, M. Activation of olefin metathesis complexes containing unsymmetrical unsaturated N-heterocyclic carbenes by copper and gold transmetalation. Chem. Commun. 2019, 55, 11583-11586. [CrossRef] [PubMed] 